\title{
Diversity Among Ralstonia solanacearum Strains Isolated from the Southeastern United States
}

\author{
Jason C. Hong, David J. Norman, David L. Reed, M. Timur Momol, and Jeffrey B. Jones
}

First author: Plant Pathology Department, University of Florida, Institute of Food and Agricultural Sciences (IFAS), Gainesville, 32611; second author: Department of Plant Pathology, University of Florida, IFAS, Mid-Florida Research and Education Center, 2725 Binion Rd., Apopka, 32703; third author: Florida Museum of Natural History, Dickinson Hall, University of Florida, Gainesville; fourth author: Plant Pathology Department, North Florida Research and Education Center, University of Florida, Quincy 32351; and fifth author: Plant Pathology Department, University of Florida, IFAS, Gainesville.

Current address of J. C. Hong: United States Department of Agriculture-Agricultural Research Service, U.S. Horticultural Research Laboratory, 2001 S. Rock Rd., Fort Pierce, FL 34945.

Accepted for publication 5 June 2012.

\section{ABSTRACT}

Hong, J. C., Norman, D. J., Reed, D. L., Momol, M. T., and Jones, J. B. 2012. Diversity among Ralstonia solanacearum strains isolated from the southeastern United States. Phytopathology 102:924-936.

This is the first comprehensive study of a collection of Ralstonia solanacearum strains from the southeastern United States to be characterized based on biovar, pathogenicity, hypersensitive reaction on tobacco, and phylogenetic analyses of the $e g l$ sequence. Rigorous phylogenetic analysis of the commonly used $e g l$ gene produced robust phylogenies that differed significantly from a neighbor-joining tree differed from and previously published phylogenies for $R$. solanacearum strains. These robust trees placed phylotype IV within the phylotype I clade, which may suggest that phylogenies based solely on egl may be misleading. As a result of phylogenetic analyses in this study, we determined that U.S. strains from Georgia, North Carolina, South Carolina, and older Florida strains isolated from solanaceous crops all belong to phylotype II sequevar 7 . However, many strains recently isolated in Florida from tomato and other crops were more diverse than the southeastern United States population. These unique Florida strains grouped with strains mostly originating from the Caribbean and Central America. One of the exotic strains, which in a previous study was determined to be established in northern Florida, was characterized more extensively. Upon using Musa-specific multiplex polymerase chain reaction, this strain produced a unique banding pattern, which has not previously been reported. Inoculation of this strain into Musa spp. did not result in wilt symptoms; however, the plants were stunted and root masses were significantly reduced. Furthermore, following root inoculation, the bacterium, unlike a typical Florida race 1 biovar 1 strain, was recovered from the roots and stems, indicating systemic movement. This is the first report of an $R$. solanacearum strain isolated in the United States that is deleterious to the growth of Musa plants.
Bacterial wilt, caused by the bacterial pathogen Ralstonia solanacearum, is one of the most destructive bacterial plant diseases in the tropical, subtropical, and temperate regions of the world. $R$. solanacearum, as a group, is known to infect over 200 different plant species but, more importantly, can cause serious yield losses to important agricultural crops such as tomato, potato, pepper, eggplant, tobacco, banana, and geranium (Pelargonium) (19). Once the bacterium is introduced into any of these production systems, losses can be extensive. In the southeastern United States, yield loss in field tomato production can be $\geq 80 \%$ when weather conditions are conducive for disease development. Although $R$. solanacearum is perceived as a tropical or subtropical pathogen, strains in the subgroup race 3 biovar 2 are known to cause bacterial wilt on potato at low temperatures (61). As a result of the potential economic impact and yield loss these strains could cause in potato production in the temperate regions of the United States, $R$. solanacearum race 3 biovar 2 is listed as a select agent in the United States under the Agricultural Bioterrorism Protection Act of 2002 (18).

Classification of $R$. solanacearum has been highly debated for more than a century. In the past 20 years, the bacterium has been

Corresponding author: J. B. Jones; E-mail address: jbjones@ufl.edu

* The $\boldsymbol{e}$-Xtra logo stands for "electronic extra" and indicates that the online version contains four supplemental figures. Figures 1 and 5 appear in color online.

http://dx.doi.org/10.1094/PHYTO-12-11-0342

(C) 2012 The American Phytopathological Society classified in three different genera. Previously, the bacterium was briefly placed in the genus Burkholderia but, due to phenotypic and genotypic differences, it was transferred to the new genus Ralstonia (68). Although $R$. solanacearum strains share common traits, groups within the species have differential characteristics such as host range, optimal temperature for causing disease symptoms, and utilization of an array of carbohydrates. These differences prompted many researchers to classify bacterial strains to the subspecies or intraspecies level based on biovar determination (19), host range (2), restriction fragment length polymorphism (RFLP) analysis (5), and phylogenetic analysis of specific genes (13).

Phylogenetic analysis has made it possible to determine the diversity of strains quickly and to a degree of accuracy. Previously, various techniques could take up to a month for definitive results (i.e., host tests or biovar test), or the tests were difficult to analyze (i.e., amplified fragment length polymorphisms, RFLP, or pulsed-field gel electrophoresis). Within the past few years, many studies have been published using DNA sequence-based phylogenetic analyses to determine the diversity of $R$. solanacearum strains $(3,22,25,26,30,32,53,62,64,65,67)$.

Multilocus sequence analysis (MLSA), which is phylogenetic analysis of multiple housekeeping genes, was previously performed on a diverse collection of $R$. solanacearum strains $(4,66)$. MLSA has revealed various recombination events, probable geographic origins of the bacterium, and identified which populations are continuing to diversify. MLSA is useful in resolving the relationship of an entire species; however, it is unable to 
discriminate sufficiently between microvariations within the population (i.e., infrasubspecies or infrasubspecific groups) (34, $36,69)$. Thus, one of the limiting factors of MLSA is that important or interesting groups of strains (e.g., the race 3 biovar 2 strains) cannot be resolved from other strains within the same subspecies $(4,66)$. In the current $R$. solanacearum hierarchical classification scheme, one sequevar (the taxonomic equivalent to infrasubspecies) groups all of the race 3 biovar 2 strains in a clade which has been designated sequevar 1 (13).

Based on traditional methods for infrasubspecies classification of $R$. solanacearum, strains originating from the southeastern United States have been identified as race 1 biovar $1(26,35$, 37,51). Although these strains appear to be similar, diversity and variation have been observed upon comparing host range, disease severity, and genotypic markers (27,51). Robertson et al. (52) observed that strains originating from the Carolinas caused wilt symptoms in tobacco, whereas a hypersensitive reaction (HR) was observed when the plants were inoculated with strains originating in Florida. They determined that strains from the Carolinas contained a truncated, nonfunctional avirulence (avr) gene, not found in the Georgia and Florida strains, which had the full-length functional avr gene (avrA). Strains with the truncated $a v r$ gene also had a broader host range, including tobacco, whereas strains with the avrA caused an HR in tobacco.

Exotic $R$. solanacearum strains recently have been identified in northern, central, and southern Florida (22,26,39). Based on multiplex polymerase chain reaction (PCR) and phylogenetic analysis of the $e g l$ gene, it is assumed that these strains have originated from Asia or the Caribbean. These exotic strains could have a wider host range than the native Florida strains. It is believed that many of the recent bacterial wilt outbreaks may have been caused by these exotic strains instead of the native strains (39). Bacterial wilt on pepper was previously reported in Florida (26). Typical $R$. solanacearum strains from the United States do not cause bacterial wilt on pepper. Using multiplex PCR and phylogenetic analysis of $e g l$, it was concluded that this strain might have Asiatic origins.

After conducting a 2-year study, in 2008 we reported on an exotic strain found in the waterways in northern Florida (22). During the 2-year sampling, typical northern Florida $R$. solanacearum strains were never detected. Strains collected in the study were compared by phylogenetic analysis of the egl sequence and pulse-field gel electrophoresis. Based on previous research, phylogenetic analysis of the $e g l$ gene of this strain showed that it was $100 \%$ identical to new emerging strains reported in Martinique (65). In both articles, it was reported that this strain was more aggressive when compared with the typical native strain. Furthermore, new strains found in Martinique, although not race 2, were able to colonize and move systemically in triploid banana without causing symptoms. The Martinique bacterial strain also had a greater host range than typical strains isolated on Martinique. In our study, we present data showing that this exotic strain not only colonized triploid banana but also was deleterious to the growth of the plant.

The objectives of this study were to (i) characterize $R$. solanacearum strains isolated in the southeastern United States, (ii) determine the genetic diversity of these strains using the hierarchical classification scheme as outlined by Fegan and Prior (13), and (iii) further characterize an emerging $R$. solanacearum strain. Extensive analysis was performed on collected strains. Strains were characterized based on biovar, pathogenicity tests, HR on tobacco, and phylogenetic analyses of $e g l$ sequences.

\section{MATERIALS AND METHODS}

Bacterial culture, biovar and inoculum preparation. All of the $R$. solanacearum strains used in this study are listed in Table 1. Upon receiving bacterial cultures, strains were streaked on modified semi-selective medium (SMSA) (10), transferred to casamino acid peptone glucose (CPG) agar (55), and then stored at $-80^{\circ} \mathrm{C}$ in a $30 \%$ glycerol solution. Atypical colonies were tested with $R$. solanacearum-specific immunoassay strips (Agdia, Inc., Elkhart, IN). All strains that were negative for the immunoassay strips twice were discarded. In total, 147 strains were collected from different libraries: 100 from Florida, 33 from the Carolinas, and 14 from Georgia. These strains were confirmed as $R$. solanacearum, because they all produced a 281-bp product when tested with universal $R$. solanacearum primers $759 / 760$ (40).

Biovar was determined based on the bacterium's ability to utilize various carbon sources, as described by Hayward (19). RS5, race 1 biovar 1, GMI1000; race 1 biovar 3; and UW447, race 3 biovar 2T, were used as standards and positive controls for the biovar tests. A bacterial culture grown on CPG for $12 \mathrm{~h}$ was suspended in sterile tap water and adjusted to approximately $10^{8}$ $\mathrm{CFU} / \mathrm{ml}$ with a spectrophotometer (Sigma-Aldrich Co., Milwaukee, WI). The suspension was added to plates containing the panel of carbon sources (dextrose, D-mannitol, sorbitol, trehalose, dulcitol, and lactose) and media without a carbon source, which served as a negative control.

For all pathogenicity tests, each strain was grown on CPG agar for $24 \mathrm{~h}$ and then suspended in sterile deionized water. Suspensions were adjusted to $10^{8} \mathrm{CFU} / \mathrm{ml}$. Plants were inoculated as described below. A few wilted plants from each experiment were selected to perform Koch's postulates. Stem segments were soaked in $70 \%$ ethanol and then flame sterilized. The plant material was crushed using a mortar and pestle. The residue was suspended in $1 \mathrm{ml}$ of deionized water and the suspension was streaked on SMSA. The plates were stored at $28^{\circ} \mathrm{C}$. R. solanacearum colonies were confirmed by PCR using primers set $759 / 760$ (40). The specific details for PCR amplification are described later in the article.

Pathogenicity tests on tomato and pepper and HR on tobacco. Pathogenicity tests were performed using tomato ('Bonny Best') and pepper ('Aruba') plants. Strains were inoculated on 3to 4 week-old plants in 10 -cm-diameter pots. Root inoculation consisted of wounding the roots and pouring $30 \mathrm{ml}$ of a $10^{8}$ $\mathrm{CFU} / \mathrm{ml}$ bacterial suspension or sterile deionized water on the surrounding soil in close proximity to the crown of the plant. The inoculated plants were transferred to a growth room with a 12-h light-and-dark cycle and the air temperature was maintained at $30^{\circ} \mathrm{C}$. Each trial consisted of three plants per strain, and was repeated twice. For the tobacco HR assay, $10^{7} \mathrm{CFU} / \mathrm{ml} R$. solanacearum suspensions were infiltrated into the intercellular space of fully expanded tobacco leaves. Each strain was inoculated twice on the same leaf and inoculations were repeated on three other plants. Inoculated plants were placed in a growth chamber with conditions previously described. Infiltrated areas were assessed for HR $24 \mathrm{~h}$ after infiltration.

PCR protocols. Each PCR amplification reaction contained 1 unit of Taq DNA polymerase (GoTaq Flexi DNA Polymerase; Promega Corp., Madison, WI) with $5.0 \mu \mathrm{l}$ of $5 \times$ buffer, $1.5 \mu \mathrm{l}$ $(25 \mu \mathrm{M})$ of $\mathrm{MgCl}_{2}, 1.0 \mu \mathrm{l}(10 \mu \mathrm{M})$ of each dNTP, each primer at $10 \mathrm{pmol}$, and $100 \mathrm{ng}$ of DNA. The total volume was adjusted to $25 \mu \mathrm{l}$ with sterile deionized water. A hot start of $95^{\circ} \mathrm{C}$ for $5 \mathrm{~min}$; followed by 30 cycles of $95^{\circ} \mathrm{C}$ for $45 \mathrm{~s}, 68^{\circ} \mathrm{C}$ for $30 \mathrm{~s}$, and $72^{\circ} \mathrm{C}$ for $60 \mathrm{~s}$; and a 10 -min extension at $72^{\circ} \mathrm{C}$ in the last cycle was used for the amplification of a DNA sequence in a thermocycler (Bio-Rad, Hercules, CA). The annealing temperature was adjusted according to the composition of the oligonucleotide sequence.

Each strain was tested to determine phylotype and to ensure that it was not race 3 biovar 2, because these strains are listed in the United States as a bioterrorism Select Agent (18). Phylotype classification, as outlined by Fegan and Prior (13), consisted of PCR using the primers Nmult21:1F, Nmult21:2F, Nmult23:AF, 
TABLE 1. List of strains characterized in this study

\begin{tabular}{|c|c|c|c|c|c|c|c|c|c|}
\hline \multirow[b]{2}{*}{ Strains ${ }^{b}$} & \multirow[b]{2}{*}{ Origin } & \multirow[b]{2}{*}{ Host origin } & \multirow[b]{2}{*}{ Other name } & \multirow[b]{2}{*}{ Year } & \multirow[b]{2}{*}{ Biovarc $^{c}$} & \multirow[b]{2}{*}{ Phylotype/sequevar ${ }^{\mathrm{d}}$} & \multicolumn{3}{|c|}{ Pathogenicity test $\mathrm{e}^{\mathrm{e}}$} \\
\hline & & & & & & & Tomato & Pepper & $\mathrm{HR}^{\mathrm{f}}$ \\
\hline RS5 & Florida & Tomato & $\ldots$ & 1999 & 1 & $\mathrm{II} / 7$ & + & + & + \\
\hline 102 & Florida & Pond water & $\ldots$ & 2004 & 1 & II & + & - & - \\
\hline 103 & Florida & Pond water & $\ldots$ & 2004 & 1 & II & + & - & - \\
\hline 104 & Florida & Pond water & $\ldots$ & 2004 & 1 & II & + & - & - \\
\hline 105 & Florida & Pond water & $\ldots$ & 2004 & 1 & II/UD & + & - & - \\
\hline 106 & Florida & Pond water & $\ldots$ & 2004 & 1 & II & + & - & - \\
\hline $107 \bullet$ & Florida & Polygonum pennsylvanicum & $\ldots$ & 2004 & 1 & II/UD & + & - & - \\
\hline 108 & Florida & P. pennsylvanicum & $\ldots$ & 2004 & 1 & II/UD & + & - & - \\
\hline 109 & Florida & P. pennsylvanicum & $\ldots$ & 2004 & 1 & II & + & - & - \\
\hline 110 & Florida & Hydrocotyle ranunculoides & $\ldots$ & 2004 & 1 & II/UD & + & - & - \\
\hline 111 & Florida & H. ranunculoides & $\ldots$ & 2004 & 1 & II & + & - & - \\
\hline 112 & Florida & H. ranunculoides & $\ldots$ & 2004 & 1 & II & + & - & - \\
\hline 113 & Florida & Pond water & $\ldots$ & 2004 & 1 & II & + & - & - \\
\hline 114 & Florida & Pond water & $\ldots$ & 2004 & 1 & II & + & + & - \\
\hline 118 & Florida & Pond water & $\ldots$ & 2004 & 1 & II/UD & + & - & - \\
\hline 119 & Florida & Pond water & $\ldots$ & 2004 & 1 & II/UD & + & - & - \\
\hline 120 & Florida & Pond water & $\ldots$ & 2004 & 1 & II/UD & + & - & - \\
\hline 125 & Florida & Pond water & $\ldots$ & 2004 & 1 & II/UD & + & - & - \\
\hline 128 & Florida & Pond water & $\ldots$ & 2004 & 1 & II/UD & + & - & - \\
\hline 130 & Florida & Pond water & $\ldots$ & 2004 & 1 & II/UD & + & - & - \\
\hline $201 \sim$ & Florida & Pothos & P487 & 1996 & 1 & II & + & - & + \\
\hline $202 \sim$ & Florida & Tomato & P503 & NA & 1 & II & + & - & - \\
\hline $203 \sim$ & Florida & Tomato & P504 & NA & 1 & $\mathrm{II} / 7$ & + & - & + \\
\hline 204 & Florida & Tomato & P505 & NA & 1 & $\mathrm{II} / 7$ & - & - & + \\
\hline 205 & Florida & Tomato & P507 & 1996 & 1 & II/4 & + & - & + \\
\hline $206 \sim$ & Florida & Tomato & P530 & 1997 & 1 & $\mathrm{II} / 7$ & + & - & + \\
\hline 207 g & Florida & Tomato & P532 & 1997 & 1 & II & - & - & + \\
\hline 208 & Florida & Tomato & P533 & 1997 & 1 & II & + & - & + \\
\hline 209 & Florida & Tomato & P534 & 1997 & 1 & II & + & - & + \\
\hline $210 \sim^{g}$ & Florida & Tomato & P535 & 1997 & 1 & II $/ 5$ or 6 & - & - & + \\
\hline $211 \sim$ & Florida & Tomato & P536 & 1997 & 1 & $\mathrm{II} / 7$ & + & - & + \\
\hline $212 \sim g$ & Florida & Potato & P541 & 1997 & 1 & II & - & - & + \\
\hline $213 \sim$ & Florida & Potato & P543 & 1997 & 1 & II & + & - & + \\
\hline $214 \sim$ & Florida & Potato & P550 & 1997 & 1 & $\mathrm{II} / 7$ & + & - & + \\
\hline $215 \sim$ & Florida & Potato & P553 & 1997 & 1 & $\mathrm{II} / 7$ & + & - & + \\
\hline $216 \sim$ & Florida & $\mathrm{NA}^{\mathrm{g}}$ & P557 & 1997 & 1 & II & + & - & - \\
\hline 217 & Florida & Pothos & P564 & 1996 & 1 & II & + & + & + \\
\hline 218 & Florida & Pothos & P573 & 1999 & 1 & II & + & - & + \\
\hline $219 \sim$ & Florida & Tomato & P576 & 1999 & 1 & II/7 & + & - & + \\
\hline $220 \sim$ & Florida & Tomato & P594 & 2000 & 1 & $\mathrm{II} / 4$ & + & - & - \\
\hline 221 & Florida & $\mathrm{NA}^{\mathrm{g}}$ & P136 & 1987 & 1 & II/7 & + & - & + \\
\hline 222 & Florida & Heliconia & $\mathrm{P} 158$ & 1988 & 1 & II $/ 5$ or 6 & + & - & + \\
\hline $223 \sim$ & Florida & Pothos & P485 & 1996 & 1 & II & + & + & + \\
\hline $224 \sim$ & Florida & Pothos & P514 & 1996 & 1 & II & + & + & + \\
\hline $225 \sim$ & Florida & Pothos & P517 & 1996 & 1 & $\mathrm{II} / 4$ & + & + & + \\
\hline $226 \sim$ & Florida & Pothos & P521 & 1996 & 1 & $\mathrm{II} / 4$ & + & + & + \\
\hline $227 \sim$ & Costa Rica & Physalis angulata & ATCC 33192 & NA & 1 & I & + & - & + \\
\hline 228 & Florida & Potato & P544 & 1994 & 1 & II/7 & + & - & + \\
\hline $229 \sim$ & Florida & Pothos & P545 & 1997 & 1 & $\mathrm{II} / 4$ & + & + & + \\
\hline $230 \sim$ & Florida & Pothos & P548 & 1997 & 1 & $\mathrm{II} / 4$ & + & - & + \\
\hline $231 \sim$ & Florida & Tomato & P589 & 1999 & 1 & II/7 & + & - & + \\
\hline $232 \sim$ & Martinique & Tomato & P609, MT1 & 1986 & 1 & I & + & - & - \\
\hline $233 \sim$ & Guadeloupe & Tomato & P606, a12 & 1985 & 1 & $\mathrm{II} / 7$ & + & - & + \\
\hline $234 \sim$ & Martinique & Tomato & P610, MT5 & 1987 & 1 & $\mathrm{II} / 7$ & + & - & + \\
\hline $235 \sim$ & NA & Anthurium & P618, 99.1119/1 & 1999 & 1 & $\mathrm{II} / 4$ & + & - & + \\
\hline $236 \sim$ & NA & Anthurium & P621, 99.1120/2 & 1999 & 1 & $\mathrm{II} / 4$ & + & - & + \\
\hline 237 & United States & Tomato & ATCC 11696 & 1953 & 1 & $\mathrm{II} / 7$ & + & - & + \\
\hline $238 \sim$ & Florida & Pothos & P673 & 2003 & 1 & $\mathrm{II} / 4$ & + & - & - \\
\hline 239 & North Carolina & Potato & P688, PS119 & 1945 & 1 & II/7 & + & - & + \\
\hline & & & & & & & & tinued or & tt page \\
\hline
\end{tabular}

${ }^{a} \mathrm{UD}=$ undefined sequevar and NA = information was not available.

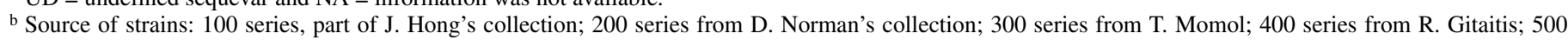
series from D. Kluepfel and A. Mila, except for 527, which is part of A. Alvarez's collection; UW from C. Allen's collection; and AW from M. Schell's collection. Noted strains were previously characterized: $\square(5), \bullet(22), \sim(39), *(42), \diamond(45), \Delta(52),{ }^{\wedge}(54),{ }^{\circ}(63)$, and $\times(70)$.

c Biovar was determined as outlined by Hayward, 1991 (20).

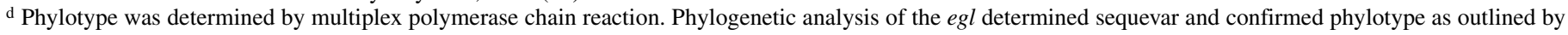
Fegan and Prior (13).

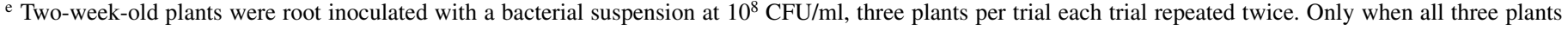
for both trials showed symptoms were the strains considered positive. Plants were stored in growth room conditions at $28^{\circ} \mathrm{C}$.

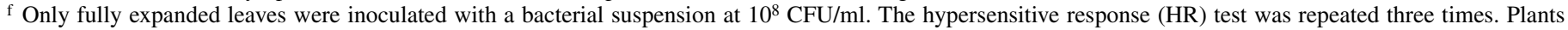
were stored in growth room conditions at $28^{\circ} \mathrm{C}$.

g Strains lost pathogenicity and appear dry and circular on agar. 


\begin{tabular}{|c|c|c|c|c|c|c|c|c|c|}
\hline \multirow[b]{2}{*}{ Strains ${ }^{b}$} & \multirow[b]{2}{*}{ Origin } & \multirow[b]{2}{*}{ Host origin } & \multirow[b]{2}{*}{ Other name } & \multirow[b]{2}{*}{ Year } & \multirow[b]{2}{*}{ Biovar ${ }^{\mathrm{c}}$} & \multirow[b]{2}{*}{ Phylotype/sequevar ${ }^{\mathrm{d}}$} & & ogenicity & \\
\hline & & & & & & & Tomato & Pepper & $\mathrm{HR}^{\mathrm{f}}$ \\
\hline 240 & North Carolina & Potato & P689, PS121 & 1945 & 1 & $\mathrm{II} / 7$ & + & - & + \\
\hline 241 & North Carolina & Tomato & P690, PS123 & 1953 & 1 & $\mathrm{II} / 7$ & + & - & + \\
\hline 242 & North Carolina & Tobacco & P691, PS124 & 1954 & 1 & $\mathrm{II} / 7$ & + & - & + \\
\hline $301^{\mathrm{g}}$ & Florida & Soil & RS14 & 2001 & 1 & II/UD & - & - & + \\
\hline $302^{\mathrm{g}}$ & Florida & Tomato & RS18 & 1991 & 1 & $\mathrm{II} / 7$ & - & - & + \\
\hline $303 \bullet$ & Florida & Geranium & RS37 & 2001 & 1 & II/UD & + & - & - \\
\hline $304 \bullet$ & Florida & Geranium & RS38 & 2001 & 1 & II/UD & + & + & - \\
\hline 305 & Florida & Geranium & RS39 & 2001 & 1 & II & + & - & - \\
\hline 306 & Florida & Geranium & RS40 & 2001 & 1 & II/UD & + & - & - \\
\hline 307 & Florida & Pond water & RS51 & 2001 & 1 & II & + & - & - \\
\hline $308 \bullet$ & Florida & Bidens mitis & RS55 & 2002 & 1 & II & + & - & - \\
\hline $309 \bullet$ & Florida & P. pennsylvanicum & RS56 & 2002 & 1 & II & + & - & - \\
\hline 310 & Florida & P. pennsylvanicum & RS57 & 2002 & 1 & II & + & - & - \\
\hline $311 \bullet$ & Florida & H. ranunculoides & RS58 & 2002 & 1 & II & + & - & - \\
\hline 312 & Florida & H. ranunculoides & RS59 & 2002 & 1 & II/UD & + & - & - \\
\hline 313 & Florida & H. ranunculoides & RS60 & 2002 & 1 & II & + & - & - \\
\hline 314 & Florida & H. ranunculoides & RS61 & 2002 & 1 & II & + & - & - \\
\hline $315 \bullet$ & Florida & Pond water & RS62 & 2002 & 1 & II & + & - & - \\
\hline $316 \bullet$ & Florida & Pond water & RS65 & 2002 & 1 & II & + & - & - \\
\hline $317 \bullet$ & Florida & B. mitis & RS66 & 2002 & 1 & II & + & - & - \\
\hline $318 \bullet$ & Florida & Pond water & RS67 & 2002 & 1 & II & + & - & - \\
\hline $319 \bullet$ & Florida & P. pennsylvanicum & RS70 & 2002 & 1 & II & + & - & - \\
\hline 320 & Florida & Pond water & RS74 & 2003 & 1 & II & + & - & - \\
\hline 321 & Florida & Pond water & RS77 & 2003 & 1 & II & + & - & + \\
\hline 322 & Florida & Pond water & RS79 & 2003 & 1 & II & + & - & - \\
\hline 323 & Florida & Pond water & RS85 & 2003 & 1 & II & + & - & + \\
\hline 324 & Florida & Pond water & RS89 & 2003 & 1 & II & + & - & - \\
\hline 325 & Florida & Pond water & RS90 & 2003 & 1 & II & + & - & + \\
\hline 326 & Florida & Pond water & RS101 & 2004 & 1 & II & + & - & - \\
\hline 327 & Florida & Pond water & RS109 & 2004 & 1 & II & + & - & + \\
\hline 328 & Florida & Hydrangea & RS116 & 2005 & 1 & II & + & - & + \\
\hline $329 \bullet$ & Florida & Hydrangea & RS118 & 2005 & 1 & II & + & - & + \\
\hline 330 & Florida & Pepper & RS121 & 2005 & 3 & I & + & + & - \\
\hline 331 & Florida & Pepper & RS122 & 2005 & 3 & I & + & + & - \\
\hline 332 & Florida & Pepper & RS123 & 2005 & 3 & I & + & + & + \\
\hline 333 & Florida & Hydrangea & RS124 & 2005 & 1 & II & + & - & - \\
\hline $334 \bullet$ & Florida & Geranium & RS125 & 2005 & 1 & II/UD & + & - & + \\
\hline 335 & Florida & Geranium & RS126 & 2005 & 1 & II & + & - & + \\
\hline 336 & Florida & Geranium & RS127 & 2005 & 1 & II & + & - & - \\
\hline $337 \bullet$ & Florida & Geranium & RS128 & 2005 & 1 & II & + & - & + \\
\hline 338 & Florida & Pond water & RS129 & 2005 & 1 & II & + & - & + \\
\hline $339 \bullet$ & Florida & Pond water & RS130 & 2005 & 1 & II & + & - & - \\
\hline 340 & Florida & Tomato & RS133 & 2005 & 1 & II & + & - & - \\
\hline 341 & Florida & Tomato & RS134 & 2005 & 1 & II & + & - & - \\
\hline 342 & Florida & Tomato & RS135 & 2005 & 1 & II & + & - & + \\
\hline 343 & Florida & Tomato & RS136 & 2005 & 1 & II & + & - & + \\
\hline 344 & Florida & Tomato & RS137 & 2005 & 1 & II & + & - & + \\
\hline 348 & North Carolina & Tobacco & TD294 & NA & 1 & II & + & - & + \\
\hline 352 & Georgia & Tomato & TD674 & $\mathrm{NA}$ & 1 & II & + & - & - \\
\hline 353 & Florida & Tomato & RS73 & 2006 & 1 & II & + & - & + \\
\hline 355 & Florida & Tomato & RS1 & 1999 & 1 & II & + & - & + \\
\hline $356^{\mathrm{g}}$ & Georgia & Tomato & RS3 & 1999 & 1 & II & - & - & - \\
\hline $357 *$ & Georgia & Tomato & UW26 & 1954 & 1 & II & + & - & - \\
\hline 359 & North Carolina & Tomato & UW123 & $\mathrm{NA}$ & 1 & II & - & - & - \\
\hline $362^{\circ}$ & North Carolina & Tobacco & UW203 & 1969 & 1 & II & + & - & - \\
\hline 363 & North Carolina & Tobacco & UW209 & 1969 & 1 & II & + & - & - \\
\hline 368 & Florida & Tomato & $\ldots$ & NA & 1 & II & + & - & + \\
\hline 369 & Florida & Tomato & $\ldots$ & NA & 1 & II & + & - & + \\
\hline 370 & North Carolina & Tobacco & $\ldots$ & NA & 1 & II & + & - & - \\
\hline 373 & Georgia & Tomato & $\ldots$ & NA & 1 & II & - & - & - \\
\hline 374 & Georgia & Tomato & 286 & NA & 1 & II & - & - & - \\
\hline 375 & Florida & Tomato & $\ldots$ & NA & 1 & II & - & - & - \\
\hline $401^{\wedge}$ & Georgia & Tomato & Rso81-5 & 1981 & 1 & II & + & - & - \\
\hline $402^{\wedge}$ & Georgia & Tobacco & Rso96-41 & 1996 & 1 & $\mathrm{II} / 7$ & + & + & - \\
\hline $403^{\wedge}$ & Georgia & Tomato & Rso81-2 & 1981 & 1 & $\mathrm{II} / 7$ & + & - & - \\
\hline 404 & Georgia & Tomato & Rso86-2 & 1986 & 1 & $\mathrm{II} / 7$ & + & - & - \\
\hline 405 & Georgia & Coffee Weed & Rso80-1 & 1980 & 1 & $\mathrm{II} / 7$ & + & + & - \\
\hline $406^{\wedge}$ & Georgia & Tomato & Rso87-105 & 1987 & 1 & II & + & - & - \\
\hline $407^{\wedge}$ & Georgia & Potato & Rso84-1 & 1984 & 1 & $\mathrm{II} / 7$ & + & + & - \\
\hline $501^{\circ}$ & North Carolina & Tobacco & K60 & $\mathrm{NA}$ & 1 & $\mathrm{II} / 7$ & + & - & - \\
\hline $502 \Delta$ & North Carolina & Tobacco & $\mathrm{NC} 116$ & NA & 1 & $\mathrm{II} / 7$ & + & - & - \\
\hline & & & & & & & & atinued or & $x t$ page \\
\hline
\end{tabular}




\begin{tabular}{|c|c|c|c|c|c|c|c|c|c|}
\hline \multirow[b]{2}{*}{ Strains $^{b}$} & \multirow[b]{2}{*}{ Origin } & \multirow[b]{2}{*}{ Host origin } & \multirow[b]{2}{*}{ Other name } & \multirow[b]{2}{*}{ Year } & \multirow[b]{2}{*}{ Biovar $^{\mathrm{c}}$} & \multirow[b]{2}{*}{ Phylotype/sequevar ${ }^{\mathrm{d}}$} & \multicolumn{3}{|c|}{ Pathogenicity test $\mathrm{e}^{\mathrm{e}}$} \\
\hline & & & & & & & Tomato & Pepper & $\mathrm{HR}^{\mathrm{f}}$ \\
\hline $503 \Delta$ & Georgia & Tobacco & GA122 & NA & 1 & $\mathrm{II} / 7$ & + & - & - \\
\hline $504 \Delta$ & Georgia & Tobacco & GA142 & NA & 1 & $\mathrm{II} / 7$ & + & - & - \\
\hline $505 \Delta$ & South Carolina & Tobacco & SC06 & NA & 1 & $\mathrm{II} / 7$ & + & - & - \\
\hline 506 & South Carolina & Tobacco & SC121 & NA & 1 & $\mathrm{II} / 7$ & + & - & - \\
\hline $507 \Delta$ & South Carolina & Tobacco & SC108 & NA & 1 & $\mathrm{II} / 7$ & + & - & - \\
\hline 508 & North Carolina & Tobacco & L-17 & NA & 1 & II/7 & + & - & - \\
\hline 509 & North Carolina & Tobacco & L-18 & NA & 1 & $\mathrm{II} / 7$ & + & - & - \\
\hline 510 & North Carolina & Tobacco & L-19 & NA & 1 & $\mathrm{II} / 7$ & + & - & - \\
\hline 511 & North Carolina & Tobacco & $\mathrm{J}-12 \mathrm{a}$ & NA & 1 & II & + & - & + \\
\hline 512 & North Carolina & Tobacco & $\mathrm{T}-24$ & NA & 1 & $\mathrm{II} / 7$ & + & - & + \\
\hline 513 & North Carolina & Tobacco & $\mathrm{T}-30$ & NA & 1 & $\mathrm{II} / 7$ & + & - & - \\
\hline 514 & North Carolina & Tobacco & $\mathrm{T}-32$ & NA & 1 & $\mathrm{II} / 7$ & + & - & - \\
\hline 515 & North Carolina & Tobacco & $\mathrm{T}-33$ & NA & 1 & $\mathrm{II} / 7$ & + & - & - \\
\hline 516 & North Carolina & Tobacco & V-167a & NA & 1 & $\mathrm{II} / 7$ & + & - & - \\
\hline 517 & North Carolina & Tobacco & $\mathrm{V}-167 \mathrm{~b}$ & NA & 1 & $\mathrm{II} / 7$ & + & - & - \\
\hline 518 & North Carolina & Tobacco & V-168 & NA & 1 & $\mathrm{II} / 7$ & + & - & - \\
\hline 519 & North Carolina & Tobacco & V-170 & NA & 1 & $\mathrm{II} / 7$ & - & - & - \\
\hline 520 & North Carolina & Tobacco & $40 \mathrm{a}$ & NA & 1 & $\mathrm{II} / 7$ & + & - & - \\
\hline 521 & North Carolina & Tobacco & K66 & NA & 1 & $\mathrm{II} / 7$ & + & - & - \\
\hline 522 & North Carolina & Tobacco & K68 & NA & 1 & $\mathrm{II} / 7$ & + & - & - \\
\hline 523 & North Carolina & Tobacco & K69 & NA & 1 & $\mathrm{II} / 7$ & + & - & - \\
\hline 524 & North Carolina & Tobacco & K71 & NA & 1 & $\mathrm{II} / 7$ & + & - & - \\
\hline $525 \square$ & North Carolina & Tomato & K74 & NA & 1 & $\mathrm{II} / 7$ & + & - & - \\
\hline $526 \square$ & North Carolina & Tomato & K136 & NA & 1 & $\mathrm{II} / 7$ & + & - & - \\
\hline $527 \times$ & Hawaii & Heliconia & A3908 & NA & 1 & II & - & - & - \\
\hline UW2 & NA & NA & $\ldots$ & NA & 1 & II & NA & NA & NA \\
\hline UW70口 & Colombia & Plantain & $\ldots$ & NA & 1 & II & NA & NA & NA \\
\hline UW170x & Colombia & Heliconia & $\ldots$ & NA & 1 & II & NA & NA & NA \\
\hline $\mathrm{AW} 1 \Delta$ & Alabama & Tomato & $\ldots$ & NA & 1 & II & + & - & + \\
\hline
\end{tabular}

Nmult22:InF, and Nmult22:RR. The PCR products electrophoresed through a $1.0 \%$ agarose gel were visualized with UV light after ethidium bromide staining. Phylotype was assigned to each strain according to band lengths. Primer pair 630/631, specific for race 3 biovar 2 strains, was used to amplify DNA from each strain (12). DNA from UW551, a race 3 biovar 2 strain, was used as a positive control.

Phylogenetic analysis of $\boldsymbol{e g l}$. The $e g l$ gene was amplified by PCR and the product was sequenced and phylogenetically analyzed to determine the relationships among new and previously described strains (Tables 1 and 2). Phylogenetic analysis was performed on a dataset consisting of 121 strains (Table 1) and then with 65 strains (Table 2), representing all of the $R$. solanacearum egl sequences submitted to GenBank, and were published in peer-reviewed journals as of June 2010, for a total of 186 strains. A complete list of the sequences acquired from GenBank can be found in Table 2. These strains originated from different regions of the world and were isolated from multiple hosts. The pepper strains were not included in this analysis. The primer pair JHFegl: GACGATGCATGCCGCTGGTCGC and JHRegl: CACGAACACCACGTTGCTCGCATTGG was designed based on the egl sequences of GMI1000 (AL646053.1), UW551 (DQ657596.1), and Molk2 (CU694393.1) accessed from GenBank. Closely related Polymorphum gilvum (CP002568.1) served as the outgroup. Amplicons were sequenced in both the forward and reverse directions to create a consensus sequence. Sequences were obtained at the University of Florida high-throughput genomic facility, Interdisciplinary Center for Biotechnology Research, using industry standard methods. Each time PCR products were submitted for sequencing, GMI1000 was resequenced to serve as a control. The sequences were aligned using Muscle (9) via http://www.ebi.ac.uk/Tools/muscle/index.html. Se-Al version 2.0a11 was used for manual manipulation of the sequences. The program was available at http://tree.bio.ed.ac.uk/software/seal/ (47). Phylogenetic analysis using maximum parsimony (MP), maximum likelihood (ML), and neighbor-joining (NJ) was run on PAUP* version 4.0b10 (57). The MP and ML trees were created by performing heuristic searches with random stepwise addition and tree bisection/reconnection (TBR) branch swapping for both analyses. Modeltest 3.7 was used to select a nucleotide model that best fit the data (43). ML settings were adjusted to the TIM+I+G nucleotide substitution model: the best-fit model suggested by Akaike's information criterion. Branch support for MP, ML, and $\mathrm{NJ}$ trees was estimated by nonparametric bootstrapping $(n=$ 1,000 replicates for MP and NJ and $n=200$ replicates for ML) by TBR swapping ( $n=1,000$ replicates for MP and NJ and $n=100$ replicates for ML) (16). RAxML-VI-HPC was used to run additional ML heuristic search and ML bootstrap analysis, which was performed on the Fisher Cluster at The University of Florida Genetics Institute, Gainesville (56). The settings in RAxML could not be adjusted to the TIM+I+G model; thus, a GTR+G model was used. For bootstrap analysis, 5,000 runs were used to generate 5,000 trees. Treefig was used to create and visualize the consensus tree (48). MrBayes 3.1 was used to perform Bayesian analysis (24). The settings were adjusted to resemble TIM+I+G and were set to run for 1,000,000 generations, being sampled at every 500th generation. The burn-in was placed at 56,000 for the smaller analysis and 88,500 for the larger, and the consensus tree of the posterior probabilities was created using PAUP* 4.0b10.

Characterization of an emerging $R$. solanacearum strain from northern Florida. Based on phylogenetic analysis of egl, RS37 was previously reported to be identical to the Martinique nonpathogenic banana strains (22). These nonpathogenic banana strains were able to colonize and move systemically in triploid banana without causing symptoms (65). To determine whether RS37 was potentially pathogenic on Musa spp., the Musa multiplex PCR protocol as described by Prior and Fegan was used (46). RS5 was used as a negative control and 527, a race 2 strain, was used as a positive control.

The Musa pathogenicity test was performed on three different Musa genotypes: 'Dwarf Nam wa' (ABB), 'French Horn' (AAB), and 'Gran Nain' (AAA). The first two genotypes were plantain and the last genotype was banana. The plants were inoculated with RS5; RS37; the race 2 strains UW2, UW70, and UW170; 
TABLE 2. List of strains obtained from GenBank used for phylogenetic analysis ${ }^{\mathrm{a}}$

\begin{tabular}{|c|c|c|c|c|c|c|}
\hline Strain & Origin & Host & Other number & Biovar & Phylotype/sequevar & $\begin{array}{c}\text { GenBank } \\
\text { accession number }\end{array}$ \\
\hline A3909 & United States & Heliconia & $\ldots$ & 1 & II/6 & EF371812.1 \\
\hline $\mathrm{ACH} 0732$ & Australia & Tomato & UW433 & 2 & $\mathrm{IV} / 7$ & GQ907150.1 \\
\hline ANT307 & FWI & Anthurium & CFBP6784 & 1 & II/4 & DQ657648.1 \\
\hline Aoyu & Australia & Potato & $\ldots$ & 2 & $\mathrm{II} / 1$ & FJ561083.1 \\
\hline $\mathrm{B} 1$ & China & Sweet potato & $\ldots$ & 4 & $\mathrm{I} / 15$ & FJ561159.1 \\
\hline Blood disease R233 & ND & ND & $\ldots$ & $\ldots$ & IV & DQ011542.1 \\
\hline CFBP734 & Madagascar & Potato & JS767 & $\ldots$ & III/19 to 23 & AF295274.1 \\
\hline CFBP765 & Japan & Tomato & JS771 & 4 & $\mathrm{I} / \mathrm{ND}$ & EF371810.1 \\
\hline CFBP1183 & Costa Rica & Heliconia & JS793 & 1 & $\mathrm{II} / 3$ & EF371805.1 \\
\hline CFBP1409 & Honduras & Musa sp. & K135, JS77 & 1 & II $/ 3$ & EF371808.1 \\
\hline CFBP2047 & United States & Tomato & $\ldots$ & 1 & II/7 & AF295262.1 \\
\hline CFBP2957 & FWI & Tomato & MT5 & 1 & II/5or36 & EF371807.1 \\
\hline CFBP2958 & FWI & Tomato & GT4 & 1 & $\mathrm{II} / 5$ & AF295266.1 \\
\hline CFBP2968 & Guadeloupe & Eggplant & RUN58 & $\ldots$ & $\mathrm{I} / 13$ to 18 & EF371806.1 \\
\hline CFBP2972 & Martinique & Tomato & RUN27 & 1 & $\mathrm{II} / 5$ & EF371809.1 \\
\hline CFBP3059 & Burkina Faso & Eggplant & JCG.AU28 & 1 & $\mathrm{III} / 23$ & AF295270.1 \\
\hline CFBP3858 & Netherlands & Potato & JS907 & 1 & $\mathrm{II} / 1$ & AF295259.1 \\
\hline CFBP6786 & Martinique & Tomato & SPV98-1537 & $\ldots$ & $\mathrm{II} / 4$ & EF371823.1 \\
\hline CFBP6801 & Martinique & Heliconia caribea & RUN279 & $\ldots$ & II & EF371836.1 \\
\hline CIP301 & Peru & Potato & $\mathrm{R} 311$ & 1 & $\mathrm{II} / 5$ & GU295003.1 \\
\hline CIP309 & Colombia & Potato & UW80, S206 & 2 & $\mathrm{II} / 2$ & EF647735.1 \\
\hline CIP312 & Peru & Eggplant & $\ldots$ & $2 \mathrm{~T}$ & II & EF647740.1 \\
\hline CIP418 & Indonesia & Peanut & MOH6 & 1 & II $/ 3$ & GU295005.1 \\
\hline Col8 & Colombia & Desmodium & $\ldots$ & $\ldots$ & $\mathrm{II} / 4 \mathrm{~PB}$ & EU795341.1 \\
\hline Col41 & Colombia & Musa sp. & $\ldots$ & $\ldots$ & II/4PB & EU795348.1 \\
\hline CMR15 & Cameroon & Tomato & $\ldots$ & $\ldots$ & III/29 & FP885896.1 \\
\hline DAR64836 & Australia & Musa sp. & $\ldots$ & 1 & $\mathrm{II} / 6$ & DQ011551.1 \\
\hline E2 & China & Eggplant & $\ldots$ & 4 & $\mathrm{I} / 15$ & FJ561157.1 \\
\hline E69 & China & Eggplant & $\ldots$ & 3 & $\mathrm{I} / 34$ & FJ561092.1 \\
\hline EU2 & China & Eucalyptus & $\ldots$ & 3 & $\mathrm{I} / 44$ & FJ561152.1 \\
\hline GMI1000 & F-Guyana & Tomato & JS771, RUN54 & 3 & $\mathrm{I} / 12$ or 18 & EF192968.1 \\
\hline ICMP6782 & Brazil & Musa sp. & $\ldots$ & $\ldots$ & $\mathrm{II} / 6$ & DQ011553.1 \\
\hline ICMP7963 & Kenya & Potato & RUN55 & $\ldots$ & II/7 & AF295263.1 \\
\hline ICMP9600 & Brazil & Musa sp. & $\ldots$ & $\ldots$ & $\mathrm{II} / 6$ & DQ011554.1 \\
\hline ICMP9915 & Indonesia & Cloves & $\ldots$ & 1 & IV & DQ011555.1 \\
\hline IPO1609 & Netherlands & Potato & RUN1 & $\ldots$ & II/ 1 and 2 & EF371814.1 \\
\hline ISBSF 1900 & Brazil & Musa sp. & RUN301 & $\ldots$ & $\mathrm{II} / 6$ & EF371839.1 \\
\hline $\mathrm{J} 25$ & Kenya & Potato & $\ldots$ & $\mathrm{N} 2$ & $\mathrm{III} / 22$ & AF295279.1 \\
\hline JT516 & Reunion Is. & Potato & RUN160 & $\ldots$ & II/1 and 2 & AF295258.1 \\
\hline JT523 & Reunion Is. & Potato & RUN333 & $\ldots$ & $\mathrm{I} / 13$ to 18 & AF295252.1 \\
\hline JT525 & Reunion Is. & Pelargonium asperum & RUN60 & $\ldots$ & III/19 to 23 & DQ657650.1 \\
\hline JT528 & Reunion Is. & Potato & $\ldots$ & 1 & III/19 & AF295273.1 \\
\hline K60 & United States & Tomato & UW25 & 1 & $\mathrm{II} / 7$ & DQ657614.1 \\
\hline LNPV19.66 & French & Potato & $\ldots$ & $\ldots$ & $\mathrm{II} / 1$ & GU295037.1 \\
\hline LNPV23.54 & France & Potato & $\ldots$ & $\ldots$ & $\mathrm{II} / 1$ & GU295038.1 \\
\hline LNPV32.36 & French Guinana & Musa sp. & $\ldots$ & $\ldots$ & $\mathrm{II} / 4$ & GU294931.1 \\
\hline LNPV32.40 & French Guinana & Musa sp. & $\ldots$ & $\ldots$ & $\mathrm{II} / 4$ & GU294933.1 \\
\hline M2 & China & Mulberry & $\ldots$ & 5 & $\mathrm{I} / 48$ & FJ561067.1 \\
\hline M3 & China & Mulberry & $\ldots$ & 5 & $\mathrm{I} / 44$ & FJ561106.1 \\
\hline M4 & China & Mulberry & $\ldots$ & 5 & $\mathrm{I} / 12$ & FJ561107.1 \\
\hline M6 & China & Mulberry & $\ldots$ & 3 & $\mathrm{I} / 48$ & FJ561109.1 \\
\hline M7 & China & Mulberry & $\ldots$ & 5 & $\mathrm{I} / 12$ & FJ561110.1 \\
\hline MAFF301558 & Japan & Potato & RUN71 & $\ldots$ & IV/8 or 10 & DQ657634.1 \\
\hline MOLK2 & Philippines & Musa sp. & $\ldots$ & $\ldots$ & II $/ 3$ & EF371841.1 \\
\hline NCPPB332 & Zimbabwe & Potato & RUN75 & $\ldots$ & III/19 to 23 & DQ657649.1 \\
\hline NCPPB3190 & Malaysia & Tomato & RUN78 & $\ldots$ & $\mathrm{I} / 13$ to 18 & AF295253.1 \\
\hline NCPPB3987 & Brazil & Potato & RUN81 & $\ldots$ & II/ND & AF295261.1 \\
\hline $\mathrm{O} 3$ & China & Olive tree & $\ldots$ & 3 & $\mathrm{I} / 44$ & FJ561069.1 \\
\hline P11 & China & Peanut & $\ldots$ & 3 & $\mathrm{I} / 17$ & FJ561068.1 \\
\hline P16 & China & Peanut & $\ldots$ & 3 & $\mathrm{I} / 18$ & FJ561114.1 \\
\hline Pe1 & China & Pepper & $\ldots$ & 3 & $\mathrm{I} / 14$ & FJ561154.1 \\
\hline Pe5 & China & Pepper & $\ldots$ & 3 & $\mathrm{I} / 34$ & FJ561091.1 \\
\hline $\mathrm{Po} 2$ & China & Potato & $\ldots$ & 2 & $\mathrm{II} / 1$ & FJ561158.1 \\
\hline Po14 & China & Potato & $\ldots$ & 2 & $\mathrm{I} / 13$ & FJ561162.1 \\
\hline Po82 & Mexico & Potato & $\ldots$ & 1 & II/4 & FJ561070.1 \\
\hline Po152 & Mexico & Potato & $\ldots$ & 3 & $\mathrm{I} / 18$ & FJ561148.1 \\
\hline Po276 & Australia & Potato & $\ldots$ & 2 & $\mathrm{II} / 1$ & FJ561082.1 \\
\hline PSI07 & Indonesia & Tomato & $\ldots$ & $\ldots$ & $\ldots$ & FP885891.1 \\
\hline PSI7 & Indonesia & Tomato & RUN83 & 2 & IV/8 or 10 & EF371804.1 \\
\hline PSS81 & Taiwan & Tomato & $\ldots$ & 3 & $\mathrm{I} / 14$ & FJ561066.1 \\
\hline PSS219 & Taiwan & Tomato & $\ldots$ & 3 & $\mathrm{I} / 34$ & FJ561167.1 \\
\hline \multicolumn{7}{|c|}{ (continued on next page) } \\
\hline
\end{tabular}

a Phylotype, sequevar, biovar, host, and origin information for the were obtained from previously published literature $(3,4,13,14,32,41,47,63,64)$; ND $=$ unknown. 


\begin{tabular}{|c|c|c|c|c|c|c|}
\hline Strain & Origin & Host & Other number & Biovar & Phylotype/sequevar & $\begin{array}{c}\text { GenBank } \\
\text { accession number }\end{array}$ \\
\hline PSS358 & Taiwan & Tomato & $\ldots$ & 3 & $\mathrm{I} / 15$ & FJ561065.1 \\
\hline $\mathrm{R} 230$ & Indonesia & Banana & $\ldots$ & $\mathrm{BDB}$ & $\mathrm{IV} / 10$ & AF295280.1 \\
\hline R288 & China & Mulberry & HT659 & 5 & $\mathrm{I} / 18$ & GQ907153.1 \\
\hline R292 & China & Mulberry & RUN91 & $\ldots$ & $\mathrm{I} / 12$ & DQ657635.1 \\
\hline $\mathrm{R} 058$ & ND & ND & $\ldots$ & ND & ND & DQ011543.1 \\
\hline RS38 & Iran & Potato & $\ldots$ & $2 \mathrm{~T}$ & II & EF647742.1 \\
\hline RS39 & Iran & Potato & $\ldots$ & $2 \mathrm{~T}$ & II & EF647743.1 \\
\hline Tb3 & China & Tobacco & $\ldots$ & 3 & $\mathrm{I} / 17$ & FJ561128.1 \\
\hline $\operatorname{Tm} 2$ & China & Tomato & $\ldots$ & 3 & $\mathrm{I} / 14$ & FJ561134.1 \\
\hline $\operatorname{Tm} 3$ & China & Tomato & $\ldots$ & 3 & $\mathrm{I} / 18$ & FJ561135.1 \\
\hline $\operatorname{Tm} 11$ & China & Tomato & $\ldots$ & 3 & $\mathrm{I} / 13$ & FJ561150.1 \\
\hline Tm13 & China & Tomato & $\ldots$ & 3 & $\mathrm{I} / 17$ & FJ561133.1 \\
\hline $\operatorname{Tm} 82$ & China & Tomato & $\ldots$ & 4 & $\mathrm{I} / 16$ & FJ561094.1 \\
\hline UW9 & Costa Rica & Heliconia & JT644 & $\ldots$ & $\mathrm{II} / 3$ & AF295257.1 \\
\hline UW21 & Honduras & Banana & R371, CIP21 & 1 & II $/ 6$ & DQ011546.1 \\
\hline UW129 & Peru & Plantain & $\ldots$ & 1 & $\mathrm{II} / 4$ & EF371811.1 \\
\hline UW160 & Peru & Plantain & $\mathrm{R} 282$ & 1 & $\mathrm{II} / 4$ & GU295051.1 \\
\hline UW162 & Peru & Musa sp. & JT648 & $\ldots$ & II $/ 4$ & AF295256.1 \\
\hline UW163 & Peru & Plantain & $\ldots$ & 1 & $\mathrm{II} / 4$ & GU295052.1 \\
\hline UW167 & Costa Rica & Banana & R283, CIP125 & 1 & $\mathrm{II} / 3$ & DQ011545.1 \\
\hline UW175 & Colombia & Plantain & $\ldots$ & 1 & $\mathrm{II} / 4$ & DQ011547.1 \\
\hline UW181 & Venezuela & Plantain & JT649, K261 & 1 & II/6 & GU295053.1 \\
\hline UW477 & Peru & Potato & RUN110 & $\ldots$ & $\mathrm{II} / \mathrm{ND}$ & DQ657604.1 \\
\hline UW551 & Kenya & Geranium & $\ldots$ & 3 & $\mathrm{II} / 1$ & DQ657596.1 \\
\hline $\mathrm{Z7}$ & China & Ginger & $\ldots$ & 4 & II/16 & FJ561142.1 \\
\hline Zo4 & Philippines & Ginger & $\ldots$ & 4 & $\mathrm{I} / 14$ & FJ561156.1 \\
\hline
\end{tabular}

and sterile deionized water. For the first experiment, eight plants of each genotype were inoculated by two different methods (root inoculation or syringe). The plants were at the three-full-leaf stage when inoculated. The root inoculation was performed as described previously. For syringe inoculation, $1 \mathrm{ml}$ of the inoculum was injected into the corm. Further Musa pathogenicity tests consisted of root inoculation only of Dwarf Nam wa (ABB) plants. Sampling consisted of dividing the plants into three parts: roots, corm to the first leaf, and from the first leaf to the top of the plant with the unfolded leaves removed. At 30 days postinoculation (dpi), plant roots were rinsed, air dried overnight, and then weighed. For each experiment, the root masses were analyzed by Tukey's range test in conjunction with an analysis of variance by the SAS System for Windows program (release 9.1; SAS, Cary, $\mathrm{NC})$.

As mentioned previously, the strains isolated from northern Florida were similar to an emerging strain in Martinique (65). The Martinique strains caused wilt symptoms on cucurbits; thus, a pathogenicity test was conducted. The test included a panel of cucurbits: watermelon (i.e., 'Sugar Baby', 'Crimson Sweet', and 'Madera'), squash (i.e., 'Taybelle', 'Senator', and 'Goldfinger'), cucumber 'Marketmore 76', and cantaloupe 'Summet'. RS37 and RS5 at $10^{8} \mathrm{CFU} / \mathrm{ml}$ were root inoculated on 3- to 4-week-old plants as described previously. Plants were grown in a growth chamber with the same conditions as described previously.

\section{RESULTS}

Phenotypic characterization of strains. Multiplex PCR, as described by Fegan and Prior (13), was performed for the 147 strains. All strains, except for three isolated from Florida, had a 372-bp band that corresponds to phylotype II. The three Florida strains, isolated from pepper, were identified as phylotype I due to the presence of a 144-bp band. The 630/631 primers, specific for race 3 biovar 2 strains, were tested on all the collected strains. None of the strains produced a band with these primers.

Strains were classified based on their ability to oxidize a panel of different carbon sources. The three pepper strains were identified as biovar 3 . These three strains were able to acidify mannitol, sorbitol, dulcitol, trehalose, lactose, and maltose. The remainder of the strains corresponded to biovar 1, which only acidified dextrose and trehalose.

After plating the strains on SMSA, it was discovered that some appeared non-mucoid with a matte surface, which is typical of nonpathogenic $R$. solanacearum strains (Table 1). All of the strains were inoculated on Bonny Best tomato plants, and 141 of 147 caused typical wilt symptoms. Wilt symptoms were observed 7 to 12 dpi for all three replicates. The six strains that did not cause symptoms were non-mucoid. A few wilted plants from each trial were sampled and the bacterial colonies isolated from the plants were streaked on SMSA. These colonies were confirmed as $R$. solanacearum by using the immunostrips.

$R$. solanacearum strains were previously isolated from wilted pepper in Florida, and were identified as biovar 3 phylotype I (26). A pathogenicity test on pepper (Aruba) was conducted. All three of the phylotype I strains wilted pepper within 7 to $10 \mathrm{dpi}$. Besides the three pepper strains, five other strains from Florida and three strains from Georgia also wilted pepper. The Florida and Georgia strains that wilted pepper were biovar 1 and phylotype II, unlike the pepper strains, which were biovar 3 and phylotype I. A few plants from each trial were sampled for $R$. solanacearum and the bacterium was confirmed using immunostrips.

An HR test on tobacco was performed because it was previously reported that Florida strains produce an HR whereas Carolina strains do not (52). An HR was observed $24 \mathrm{~h}$ after inoculation in $9.7 \%$ of the Carolina strains, $46.2 \%$ of the Georgia strains, and $58 \%$ of the Florida strains. Phylogenetic analysis grouped 66 of the Florida strains with strains originating from the Caribbean. Excluding these putatively exotic strains, the total number of strains likely to be endemic in Florida was 34. Using the adjusted total, $85.3 \%$ of the Florida strains produced a positive HR on tobacco.

Phylogenetic analyses of strains. The trees were created by four different analytical methods: MP, ML, Bayesian, and NJ (Figs. 1 to 3; Supplemental Figures 1 to 4). For both the 121- and 186-strain datasets the topology of the MP, ML, and Bayesian trees was congruent for placement of the representative strains from our library and strains acquired from GenBank. The MP, ML, and Bayesian trees were used to create a consensus tree. Two 


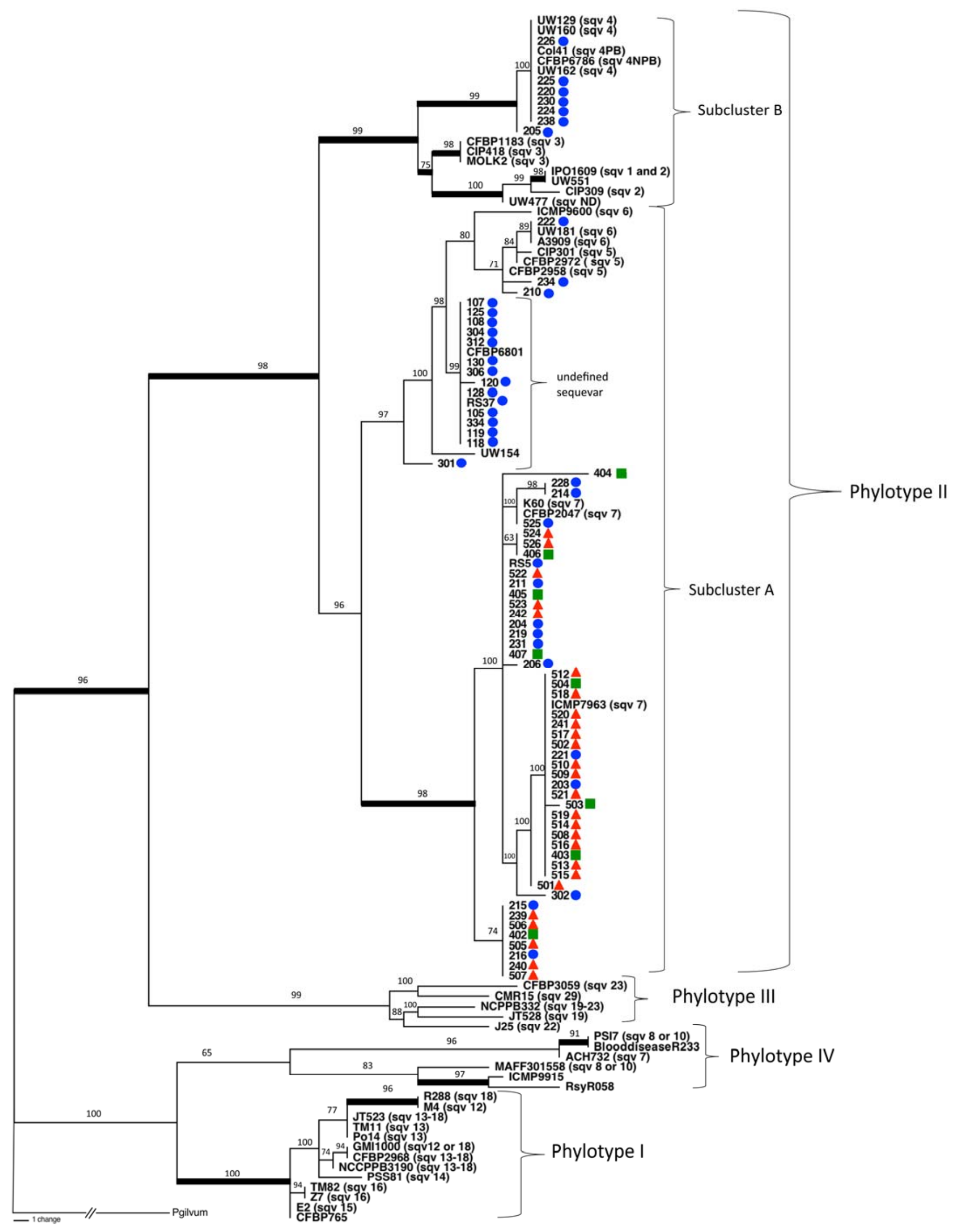

Fig. 1. Rooted tree created by Bayesian analysis of the partial egl sequences of Ralstonia solanacearum strains collected from the southeastern United States and representative strains from GenBank totaling 121 strains. The posterior probability is labeled on the branches. Weighted line indicates bootstrap value of $\geq 75 \%$ for parsimony and likelihood. Strains from Carolina are triangles, Georgia squares, and Florida circles. 
different programs were used for ML analysis, and the topologies of both of these trees were similar. The topology of the 186 strain consensus tree (Fig. 3) differed from the 121-strain consensus tree (Fig. 1), the 121-strain NJ tree (Fig. 2), and the 186-strain NJ tree. The 186-strain consensus tree placed phylotype IV within phylotype I, whereas the two phylotypes were separate for the 121strain tree and the rooted NJ tree (Fig. 3).

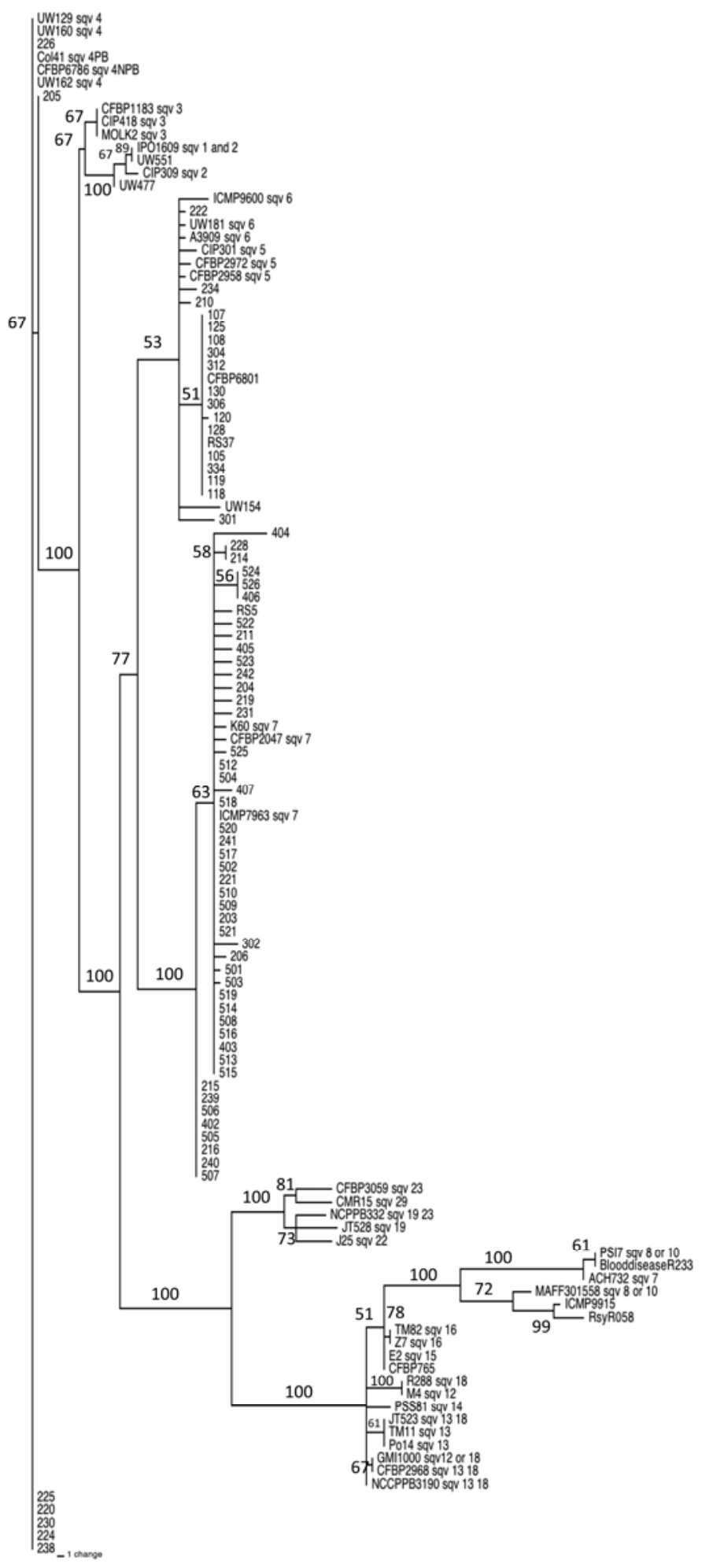

Fig. 2. Rooted phylogenetic neighbor-joining tree based partial egl sequences of 121 Ralstonia solanacearum strains collected from the southeastern United States and representative strains from GenBank. Bootstrap values indicated on the tree.
Phylogenetic analysis of $e g l$ confirmed the results from the multiplex PCR; all the strains characterized in this study were classified as phylotype II, except for the pepper strains, which were phylotype I. Strains isolated from Florida grouped with sequevars $4,5 / 6,7$, and an undefined sequevar. The undefined sequevar, located below the sequevar $5 / 6$ clade, did not contain any previously reported strains with a sequevar designation (Fig. 1). Strains isolated from the Carolinas and Georgia only grouped with sequevar 7. The majority of the older Florida strains, which were isolated prior to 1990, grouped with the sequevar 7 strains.

Characterization of an emerging $R$. solanacearum strain from northern Florida. The egl sequence of RS37 was $100 \%$ identical to a Martinique strain, CFBP6801, isolated from Heliconia caribaea. RS37 represented the strains that belonged to the undefined sequevar, which was adjacent to sequevar 5/6 (Fig. 1). Using the Musa multiplex PCR, strain 527, a Musa strain, produced a 220-bp band, which corresponded to sequevar 6 . Sequevar for strain 527 was confirmed by phylogenetic analysis of the egl sequence (Fig. 1). RS5 failed to produce any bands. RS37 produced a 167-bp band (Fig. 4). As reported, a strain that produces a 167-bp band should also produce a 351-bp band and belong to sequevar 4, like the emerging 4NPB Martinique strains $(46,65)$. However, RS37 never produced a 351-bp band. This strongly suggests that RS37 is not a 4NPB strain but a new, emerging U.S. strain. The other strains collected at the same location in Florida over a 2-year period belonged to the same clade as RS37 and also produced a 167-bp band for the Musa multiplex.

Due to the presence of a positive but unique band in RS37 with the Musa-specific primers, a triploid banana pathogenicity test was performed. Two different Musa pathogenicity tests were conducted. In the first experiment, three different Musa genotypes-Dwarf Nam wa (ABB), French Horn (AAB), and Gran Nain (AAA) - were root inoculated or inoculated injunction of the corm and pseudostem by syringe as previously described. Symptoms first appeared in the race 2 syringe-inoculated plants 7 to $10 \mathrm{dpi}$, while symptoms were observed 15 to $20 \mathrm{dpi}$ for the race 2 root-inoculated plants. The bacterium was recovered mostly in the root and corm sections in the plants root-inoculated with race 2 strains and RS37. The bacterium was recovered close to the source of inoculation or sometimes not at all for plants root inoculated with RS5. However, in plants inoculated by the syringe injection technique, RS5 and the other strains were detected in all three parts of the plants.

The root mass of the three Musa genotypes inoculated with the race 2 strains, RS5, and RS37 were weighed. The plants inoculated by the root wounding method had less root mass than those inoculated by syringe injection (figure not shown). Plants inoculated with UW2 had the lowest mass. RS37 reduced the root mass in all three genotypes; however, symptoms were more severe in Dwarf Nam wa (figure not shown). Statistical comparison of the Dwarf Nam wa root masses revealed that the root mass of the plants inoculated with RS37 was more similar to the root mass of the plants inoculated with a race 2 strain than the root mass of the plants inoculated with RS5 or the control (Fig. 5).

Wicker et al. (65) indicated that the emerging $R$. solanacearum strains had a wider host range than typical biovar 1 strains isolated from the Martinique. These strains were pathogenic on cucurbits, solanaceous crops, and ornamental plants. RS37 was inoculated on a panel of cucurbit plants to determine whether it could cause wilt symptoms. No symptoms were observed on any of the plants after 30 dpi for either RS5 or RS37.

\section{DISCUSSION}

We characterized $R$. solanacearum strains in the southeastern United States and determined that Florida had the greatest 
diversity, with strains isolated in the state belonging to two different phylotypes (22) and at least four different sequevars, whereas all strains isolated from Georgia and the Carolinas belonged to phylotype II sequevar 7. All of what are considered native strains were classified as biovar 1 and belonged to phylotype II sequevar 7. Although these strains were similar, differences were noted in HR, host range tests, and phylogenetic analysis of the $e g l$ sequence. The diversity of haplotypes found in Florida could be due to several factors. The most plausible explanation is that Florida is a subtropical region where many exotic crops are imported. The importation of these plants offers an avenue for introduction of exotic strains of the pathogen. One of the exotic strains isolated in FL, RS37, which was previously characterized $(22,26)$, was further characterized. This strain was able to cause symptoms on tomato plants like the native strain, but, more interestingly, was deleterious to growth of triploid banana. This is the first report of an $R$. solanacearum strain isolated in the United States causing symptoms in triploid banana.

Based on the phylogenetic trees created in this study, strains isolated from Florida were more diverse than those isolated in Georgia and the Carolinas. It is interesting to note that all of the strains collected from the southeastern United States from 1945 to 1987 were phylotype II sequevar 7. It is assumed that the sequevar 7 strains were endemic to the southeastern United States and, within the past 30 years, exotic strains have been introduced into this region. Yet, phylogenetic analysis was not able to differentiate between strains that caused wilt on pepper or which elicited an HR on tobacco. In a recent study, a collection of commonly used $R$. solanacearum-resistant varieties of tomato, eggplant, and pepper were challenged against 12 pathogenic strains representing phylogenetic diversity (29). The authors concluded that neither the pathoprofile (strains clustered by virulence patterns) nor the pathotypes (strains grouped based on observed phenotypic reactions) correlated to egl-based phylogeny. Therefore, it is necessary to perform both phenotypic tests and phylogenetic analysis when determining population diversity.

The majority of strains from Florida in this study grouped with sequevar 7 strains; however, a few strains grouped with sequevars 4 and 5/6. Previously, it was reported that strains belonging to sequevar 4 can infect triploid bananas and can be detected throughout the plant. Some sequevar 4 strains are true race 2 strains and can wilt triploid banana, while others are nonsystematic (65). Most of the sequevar 4 strains in this study origi- nated from pothos and anthurium cuttings. In a previous study, a Musa pathogenicity test was conducted only using 'Dwarf Cavendish' (AAA) with a few of these strains, and no symptoms were observed (39). In this study, we observed that the severity of symptoms caused by RS37 is dependent on the genotype of the banana plant. Hence, it is recommended that Musa pathogenicity tests using a variety of genotypes and the Musa multiplex PCR should be performed with all strains that group with sequevars 3 , 4 , and $5 / 6$.

Race 2 strains are described as being pathogenic on triploid banana, Heliconia spp., or both, and moderately virulent on solanaceous crops (7). The Musa multiplex PCR was created to identify race 2 strains and characterize the strains to sequevar (46). RS37 was assumed to be similar to Caribbean strains that infected triploid bananas; thus, the multiplex PCR was performed. Sequevar 4 strains will produce a 351-bp product, amplified with

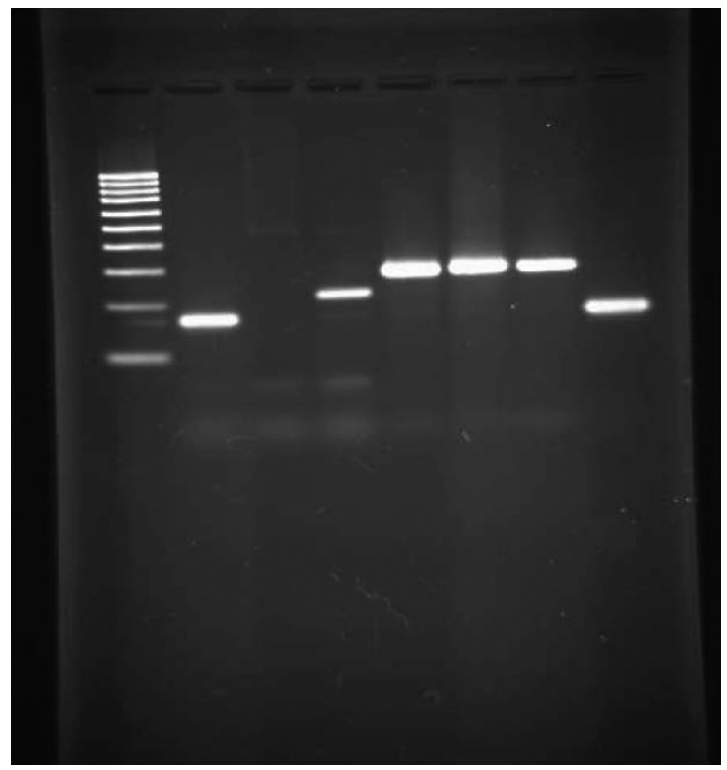

Fig. 4. Lanes 2 to 4, gel-displaying bands created by using primers for multiplex Musa; lanes 5 to 7, 759/760 Ralstonia specific primers; and lane 8, one primer set from the Musa multiplex polymerase chain reaction Musa06. Lane 1 contains ladder 10 100-bp markers. Strain RS37 was used in lanes 2, 5, and 8; RS5 in lanes 3 and 6; and 527 in lanes 4 and 7.

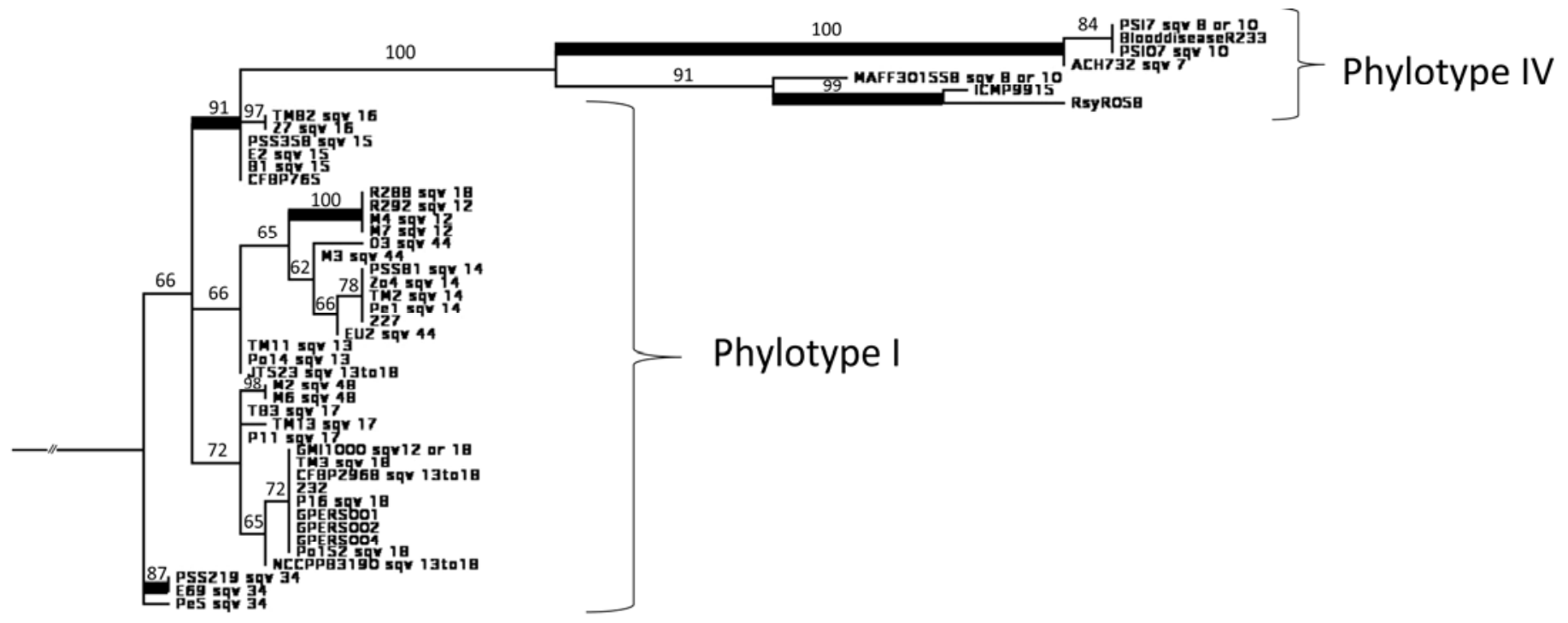

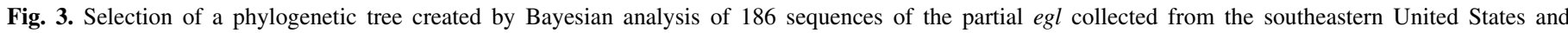

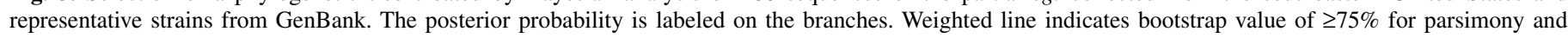
likelihood. This figure highlights the relationship of phylotype I and phylotype IV. The complete tree is Supplementary Figure 1. 
primer set Mus20, and some sequevar 4 strains will produce an additional 167-bp band amplified with primer set Mus06. Prior and Fegan (46) and Wicker (65) suggest that presence of the 167-bp amplicon might be associated with pathogenic sequevar 4
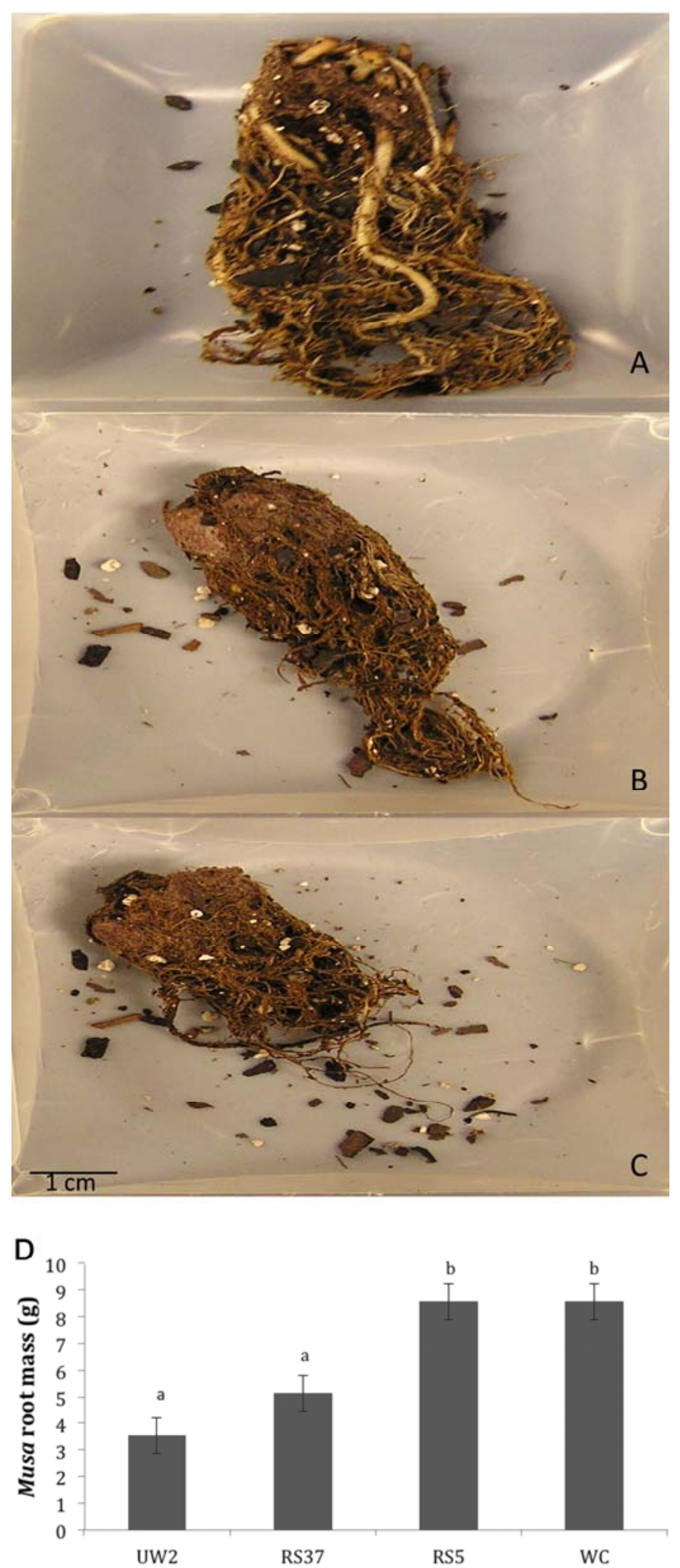

Fig. 5. Musa 'Dwarf Nam wa' root mass 30 days postinoculation air dried overnight. Roots were wounded and a $10^{8} \mathrm{CFU} / \mathrm{ml}$ suspension of A, RS5; B, RS37; and C, UW2 were poured at the crown of the plants. D, Comparison of Musa Dwarf Nam wa root mass 30 days postinoculation of plants with RS 5 (race 1), UW2 (race 2), and RS 37. Same letter over each bar indicates no significant difference according to Tukey's range test. strains. Unlike race 1, which produced no bands, RS37 produced a single 167-bp band. The 351-bp band was never observed for RS37 and other strains collected in the same region of Florida. RS37 was inoculated on a panel of Musa genotypes to determine whether it could cause symptoms. The bacterium was detected systemically on all three genotypes. The observed symptoms caused by RS37 (less root mass and reduced plant growth) were more severe on the ABB genotype than the other genotypes. RS37 is unique in that it was reported to be highly virulent on tomato $(22,26)$ and, in this study, was shown to move systemically through a banana plant. Root inoculation, as opposed to injecting the cell suspension with a hypodermic needle at the junction of the corm and the pseudostem, was the preferred technique for infecting Musa plants. With the injection technique, race 1, race 2 , and RS37 were detected in the leaves whereas, with the root inoculation technique, only race 2 and RS37 were detected in the leaves. Future research using RS37 could focus on identifying which genes are responsible for host specificity by comparing RS37 to typical race 1 and 2 strains.

In this study, using the current methods for creating phylogenetic trees, we determined that the topology of a phylogenetic tree could vary depending on the methods used for tree creation and the number of taxa used. Multiple analyses, including MP, ML, and Bayesian, were used to form a consensus tree from the outputted topologies $(33,38,71)$. By performing multiple analyses, the phylogeny was robustly analyzed and the branches are statistically supported. The tree represents all the egl sequences that were submitted from other studies to GenBank. All the strains grouped according to their previous phylotype designation. When the 186 strains were analyzed using MP, ML, and Bayesian, phylotype IV grouped within phylotype I. However, when the 121 strains were analyzed using the same methods, phylotype IV formed a separate clade from phylotype I, which is similar to previously published trees. When both datasets were analyzed by $\mathrm{NJ}$, the topology was similar to the 121-taxon tree. This could indicate that trees produced solely by $\mathrm{NJ}$ can be misleading and may not produce the most correct topology.

Constructing accurate phylogenies is dependent on type of analysis or analyses, the robustness of the program running the analysis, the quality and quantity of the data, and the quantity of taxon sampling. Many previously published articles regarding the phylogeny of $R$. solanacearum used methods of phylogenetic inference such as $\mathrm{NJ}$ and unweighted pair group method with arithmetic mean (UPGMA), or programs not designed to produce optimal trees such as the ARB software package (http://help.arbhome.de/arb.html) and Clustal W (60). These methods are often used because they produce a single phylogenetic tree rapidly for publication. However, these methods have been shown to be less effective and sometimes very misleading under certain realistic conditions $(1,15,21,28,59)$. This could explain the inconsistencies when comparing our trees to those previously published for $R$. solanacearum $(32,44,58,62)$. Inconsistent phylogenies created by inferior methods and lacking statistical supported can become the catalyst for reclassifying groups of strains. The multiple sequevar assignments of RS37 is due to inconsistent phylogenies $(22,26)$. Previous reports placed this strain and its variants in sequevar 5 and 4NPB. It appears that, due to a lack of taxon used, the incorrect sequevar was assigned, based on the tree created in this study. This strain has been assigned to an undefined sequevar because the clade did not contain any previously reported strains. Distance-based methods are inherently limited because they ignore information from insertions and or deletions, and variability within taxa are ignored $(1,11,17,59)$. Rates of evolution can vary among sites in sequences and if, during analysis, a region for a given group seems to be changing rapidly, it should affect the interpretation of the same region for a different group. The ability to propagate this information during analysis is found in likelihood and Bayesian methods and not in the distance-matrix 
methods such as UPGMA (17). In some instances, distance-treemaking methods can produce two or more trees with different topologies from the same dataset; these are called tie trees. Often, when bootstrap resampling and NJ are applied together, an arbitrary resolution of ties can produce strong support for a grouping when one does not exist (11).

Discrepancies between current and past phylogenetic reconstructions (e.g., their ability to tease apart fine-scale differences among populations) could be due to either insufficient data or incomplete taxon sampling. Unknown diversity or lack of diversity could be seen as a result of unintentionally biased sampling. One way to overcome this error is to include multiple taxa and compare analyses from different parts of the genome $(8,31,49,72)$. MLSA consists of the analysis of multiple genes found throughout the genome and is limited by not being able to resolve microvariation within the subspecies population. The accepted model to determine sequevar is sequencing and analysis of the $e g l$ gene. Yet, phylogenetic analysis of genes besides $e g l$ have yet to confirm sequevar placement. A few of these genes have shown promise in the preliminary stages but, upon using strains representing different regions of the world, the sequevar grouping was inconsistent with the topology of the egl trees (64). Clades created using cytochrome $b 561$ or mutS were similar to those produced using egl: however, a worldwide survey has yet to be performed to ascertain these genes' effectiveness $(39,65)$. Careful consideration should be made when the phylogeny of an organism is based on a single gene. DeLong and Pace (6) stated that "these extrapolations will vary in accuracy" when compared with other genes. Palleroni (41) goes so far as to say, “...phylogeny based on 16s rDNA may reflect little more than the phylogeny of the $16 \mathrm{~s}$ rDNA." In other words, phylogeny based on one gene traces the evolution of the gene and not the organism. Comparison of the genomes of $R$. solanacearum strains representing the four phylotypes revealed that phylotype I and phylotype III were very closely related and phylotype II and IV formed a clade (50). This topology is supported when comparing DNA markers throughout the genome in a forthcoming article (J. C. Hong, unpublished). Yet, phylogenetic analysis of egl indicates that phylotype I and IV are more closely related $(13,22,23,26,30,32,53,62,65)$. Comparison of complete genomes would create a phylogenetic tree that better reflects the history of the species and, hopefully, be able to group and identify populations of interest. However, due to time and cost, this method currently is unfeasible for a large survey of related organisms. It is likely that incremental improvements will be made over the previously published phylogenies, which lack certain representative strains and were analyzed with outdated phylogenetic methods.

\section{LITERATURE CITED}

1. Backeljau, T., De Bruyn, L., De Wolf, H., Jordaens, K., Van Dongen, S., and Winnepenninckx, B. 1996. Multiple UPGMA and neighbor-joining trees and the performance of some computer packages. Mol. Biol. Evol. 13:309-312.

2. Buddenhagen, I., and Kelman, A. 1964. Biological and physiological aspects of bacterial wilt caused by Pseudomonas solanacearum. Annu. Rev. Phytopathol. 2:203-230.

3. Cardozo, C., Rodíguez, P., and Marín, M. 2009. Molecular characterization of Ralstonia solanacearum species complex in the banana growing region of Uraba. Agro Colombiana 27:203-10.

4. Castillo, J. A., and Greenberg, J. T. 2006. Evolutionary dynamics of Ralstonia solanacearum. Appl. Environ. Microbiol. 73:1225-1238.

5. Cook, D. R., Barlow, E., and Sequeira, L. 1989. Genetic diversity of Pseudomonas solanacearum: Detection of restriction fragment polymorphisms with DNA probes that specify virulence and hypersensitive response. Mol. Plant-Microbe Interact. 2:113-121.

6. DeLong, E. F., and Pace, N. R. 2001. Environmental diversity of bacteria and archaea. Syst. Biol. 50:470-478.

7. Denny, T. 2006. Pages 573-644 in: Plant Pathogenic Ralstonia Species: Plant Associated Bacteria. S. S. Gnanamanickam, ed. Springer, The Netherlands.
8. Diamant, E., Palti, Y., Gur-Arie, R., Cohen, H., Hallerman, E. M., and Kashi, Y. 2004. Phylogeny and strain typing of Echerichia coli, inferred from variation at mononucleotide repeat loci. Appl. Environ. Microbiol. 70:2464-2473

9. Edgar, R. C. 2004. MUSCLE: Multiple sequence alignment with high accuracy and high throughput. Nucleic Acids Res. 32:1792-1797.

10. Englebrecht, M. C. 1994. Modification of a semi-selective medium for the isolation and quantification of Pseudomonas solanacearum. Bacterial Wilt Newsl. 10:2-5.

11. Farris, J, S., Albert, V. A., Källersjö, M., Lipscomb, D., and Kluge, A. G. 1996. Parsimony jackknifing outperforms neighbor-joining. Cladistics 12:99-124.

12. Fegan, M., Holoway, G., Hayward, A. C., and Timmis, J. 1998. Development of a diagnostic test based on the polymerase chain reaction to identify strains of solanacearum exhibiting the biovar 2 genotype. Pages 34-43 in: Bacterial Wilt Disease: Molecular and Ecological Aspects. P. Prior, C. Allen, and J. Elphinstone, eds. Springer-Verlag, Berlin.

13. Fegan, M., and Prior, P. 2005. How complex is the Ralstonia solanacearum species complex? Pages 449-461 in: Bacterial Wilt: The Disease and the Ralstonia solanacearum Species Complex. C. Allen, P. Prior, and A. C. Hayward, eds. American Phytopathological Society, St. Paul, MN.

14. Fegan, M., and Prior, P. 2006. Bacterial wilts of banana caused by members of the Ralstonia solanacearum species complex. Australas. Plant Pathol. 35:93-101.

15. Felsenstein, J. 1978. Cases in which parsimony or compatibility methods will be positively misleading. Syst. Zool. 27:401-410.

16. Felsenstein, J. 1985. Confidence limits on phylogenies: an approach using the bootstrap. Evolution 39:783-791.

17. Felsenstein. J. 2004. Inferring Phylogenies. Sinauer Associates, Inc., Sunderland, MA.

18. Hawks, B. 2002. Agricultural Bioterrorism Protection Act of 2002; Listing of Biological Agents and Toxins and Requirements and Procedures for Notification of Possession. http://www.fas.org/sgp/news/ 2002/08/fr081202.html

19. Hayward, A. C. 1964. Characteristics of Pseudomonas solanacearum. J. Appl. Bacteriol. 27:265-277.

20. Hayward, A. C. 1991. Biology and epidemiology of bacterial wilt caused by Pseudomonas solanacearum. Annu. Rev. Phytopathol. 29:65-87.

21. Hilis, D. M., Huelsenbeck, J. P., and Cunningham, C. W. 1994. Application and accuracy of molecular phylogenies. Science 264:671677.

22. Hong, J. C., Momol, M. T., Jones, J. B., Ji, P., Olson, S. M., Allen, C., Sanches Perez, A., Pradhanang, P., and Guyen, K. 2008. Detection of Ralstonia solanacearum in irrigation ponds and aquatic weeds associated with the ponds in north Florida. Plant Dis. 92:1674-1682.

23. Horita, M., Suga, Y., Ooshiro, A., and Tsuchiya, K. 2010. Analysis of genetic and biological characters of Japanese potato strains of Ralstonia solanacearum. J. Gen. Plant Pathol. 76:196-207.

24. Huelsenbeck, J. P., Ronquist, F., Nielsen, R., and Bollback, J. P. 2001. Bayesian inference of phylogeny and its impact on evolutionary biology. Science 294:2310-2314.

25. Jeong, Y., Kim, J., Kang, Y., Lee, S., and Hwang, I. 2007. Genetic diversity and distribution of Korean isolates of Ralstonia solanacearum. Plant Dis. 91:1277-87.

26. Ji, P., Allen, C., Sanchez Perez, A., Yao, J., Elphinstone, J. G., Jones, J. B., and Momol, M. T. 2007. New diversity of Ralstonia solanacearum strains associated with vegetable and ornamental crops in Florida. Plant Dis. 91:195-203.

27. Kelman, A., and Person, L. H. 1961. Strains of Pseudomonas solanacearum differing in pathogenicity to tobacco and peanut. Phytopathology 51:158-161.

28. Kuhner, M. K., and Felsenstein, J. 1994. A simulation comparison of phylogeny algorithms under equal and unequal evolutionary rates. Mol. Biol. Evol. 11:459-468 (Erratum 12:525, 1995).

29. Lebeau, A., Daunay, M.-C., Frary, A., Palloix, A., Wang, J.-F., Dintinger, J., Chiroleu, F., Wicker, E., and Prior, P. 2011. Bacterial wilt resistance in tomato, pepper, and eggplant: Genetic resources respond to diverse strains in the Ralstonia solanacearum species complex. Phytopathology 101:154-165.

30. Lewis Ivey, M. L., McSpadden Gardner, B. B., Opina, N., and Miller, S. A. 2007. Diversity and geographic distribution of Ralstonia solanacearum from eggplant in the Philippines. Phytopathology 97:1467-1475.

31. Liò P, and Goldman, N. 1998. Models of molecular evolution and phylogeny. Genome Res. 8:1233-1244.

32. Liu, Y., Kanda, A., Yano, K., Kiba, A., Hikichi, Y., Aino, M., Kawaguchi, A., Mizoguchi, S., Nakaho, K., Shiomi, H., Takikawa, Y., and Ohnishi, K. 2009. Molecular typing of Japanese strains of Ralstonia solanacearum in relation to the ability to induce a hypersensitive reaction in tobacco. J. 
Gen. Plant Pathol. 75:369-380.

33. Ludwig, W., Strunk, O., Klugbauer, S., Klugbauer, N., Weizenegger, M., Neumaier, J., Bachleitner, M., and Schleifer, K. H. 1998. Bacterial phylogeny based on comparative sequence analysis. Electrophoresis 19:554-568.

34. Maiden M. C. J. 2006. Multilocus sequence typing of bacteria. Annu. Rev. Microbiol. 60:561-588.

35. Martin, C., French, E. R., and Nydegger, U. 1982. Strains of Pseudomonas solanacearum affecting Solanaceae in the Americas. Plant Dis. 66:458-460.

36. Mathema, B., Mediavilla, J. R., Chen, L., and Kreiswirth, B. N. 2009. Evolution and taxonomy of staphylococci. Pages 31-64 in: Staphylococci in Human Disease. K. Crossley, K. Jefferson, G. Archer, and V. Fowler, eds. Wiley-Blackwell, Hoboken, NJ.

37. McLaughlin, R. J., and Sequeira, L. 1989. Phenotypic diversity in strains of Pseudomonas solanacearum isolated from a single potato field in north eastern Florida. Plant Dis. 73:960-964.

38. Newton, R. J., Jones, S. E., Eiler, A., McMahon, K. D., and Berilsson, S. 2011. A guide to the natural history of freshwater lake bacteria. Microbiol. Mol. Biol. Rev. 75:14-49.

39. Norman, D. J., Zapata, M., Gabriel, D. W., Duan, Y. P., Yuen, J. M. F., Mangravita-Novo, A., and Donahoo, R. S. 2009. Genetic diversity and host range variation of Ralstonia solanacearum entering North America. Phytopathology 99:1070-1077.

40. Opina, N., Tavner, F., Hollway, G., Wang, J. F., Li, T. H., Maghirang, R., Fegan, M., Hayward, A. C., Krishnapillai, V., and Hong, W. F. 1997. A novel method for development of species and strain-specific DNA probes and PCR primers for identifying Burkholderia solanacearum (formerly Pseudomonas solanacearum). Asia-Pac. J. Mol. Biol. Biotechnol. 5:1:1930.

41. Palleroni, N. J. 2003. Prokaryote taxonomy of the 20th century and the impact of studies on the genus Pseudomonas: A personal view. Microbiology 149:1-7.

42. Pastrik, K.-H. Elphinstone, J. G. and Pkall, R. Sequence analysis and detection of Ralstonia solanacearum by multiplex PCR amplification of 16s-23s ribosomal intergenic spacer region with internal positive control. Eur. J. Plant Pathol. 108:831-842.

43. Posada, D., and Crandall, K. A. 1998. Modeltest: Testing the model of DNA substitution. Bioinformatics 14:817-818.

44. Poussier, S., Trigalet-Demery, D., Vandewalle, P., Goffinet, B., Luisetti, J., and Trigalet, A. 2000. Genetic diversity of Ralstonia solanacearum as assessed by PCR-RFLP of the hrp gene region, AFLP and 16s rRNA sequence analysis, and identification of an African subdivision. Microbiology 146:1679-1692.

45. Pradhanang P. M., and Momol, M. T. 2001 Survival of Ralstonia solanacearum in soil under irrigated rice cultures and aquatic weeds. J. Phytopathol. 149:707-711.

46. Prior, P., and Fegan, M. 2005. Diversity and molecular detection of Ralstonia solanacearum race 2 strains by multiplex PCR. Pages 405-414 in: Bacterial Wilt Disease and the Ralstonia solanacearum Species Complex. C. Allen, P. Prior, and A. C. Hayward, eds. APS Press, St. Paul, MN.

47. Rambaut A. 1996. Se-Al v2.0a11: Sequence Alignment Editor. http://evolve.zoo.ox.ac.uk/

48. Rambaut A. 2006. FigTree v1.3.1. http://evolve.zoo.ox.ac.uk/

49. Rannala, B., Huelsenbeck, J. P., Yang, Z., and Nielsen, R. 1998. Taxon sampling and accuracy of large phylogenies. Syst. Biol. 47:702-710.

50. Remenant, B., Coupat-Goutaland, B., Guidot, A., Cellier, G., Wicker, E., Allen, C., Fegan, M., Pruvost, O., Elbaz, M., Calteau, A., Salvignol, G., Mornico, D., Mangenot, S., Barbe, V., Mèdigue, C., and Prior, P. 2010. Genomes of three tomato pathogens within the Ralstonia solanacearum species complex reveal significant evolutionary divergence. BMC Genomics 11:379.

51. Roberston, A. E., Fortnum, B. A., Wood, T. C., and Kluepfel, D. A. 2001. Diversity of Ralstonia solanacearum in the southeastern United States. Beitr. Tabakforsch. Int. 19:323-331.

52. Robertson, A. E., Wechter, W. P., Denny, T. P., Fortnum, B. A., and Kluepfel, D. A. 2004. Relationship between avirulence gene (avrA) diversity in Ralstonia solanacearum and bacterial wilt incidence. Mol.
Plant-Microbe Interact. 17:1376-1384.

53. Sanchez Perez, A., Mejia, L., Fegan, M., and Allen, C. 2008. Diversity and distribution of Ralstonia solanacearum strains in Guatemala and rare occurrence of tomato fruit infection. Plant Pathol. 57:320-331.

54. Schaad, N. W., Gaush, P. E., and Ozakman, M. 2007. Real-time PCR primers and probes for identification of Ralstonia solanacearum race 3, biovar 2 in potato and other plants. United States Patent. Patent no. $7,262,010 \mathrm{~B} 2$.

55. Schaad, N. W., Jones, J. B., and Chun, W. 2001. Laboratory Guide for Identification of Plant Pathogenic Bacteria. American Phytopathological Society Press, St. Paul, MN.

56. Stamatakis, A., Ludwig, T., and Meier, H. 2005. Raxml-iii: A fast program for maximum likelihood-based inference of large phylogenetic trees. Bioinformation 21:456-463.

57. Swofford, D. L. 2003. PAUP*: Phylogenetic Analysis Using Parsimony (* and Other Methods), version 4.0. Sinauer Associates, Sunderland, MA.

58. Taghavi, M., Hayward, A. C., Sly, L. I., and Fegan, M. 1996. Analysis of the phylogenetic relationships of strains of Burkholderia solanacearum, Pseudomonas syzgii, and the blood disease bacterium of banana based on 16s rRNA gene sequences. Int. J. Syst. Bacteriol. 46:10-15.

59. Takezaki, N. 1998. Tie trees generated by distance methods of phylogenetic reconstruction. Mol. Biol. Evol. 15:727-737.

60. Thompson, J. D., Higgins, D. G., and Gibson, T. J. 1994. CLUSTAL W: Improving the sensitivity of progressive multiple sequence alignment through sequence weighting, position-specific gap penalties and weight matrix choice. Nucleic Acids Res. 22:4673-4680.

61. Thurston, H. D. 1963. Bacterial wilt of potatoes in Colombia. Am. Potato J. 40:381-390.

62. Toukam, G. M. S., Cellier, G., Wicker, W., Guilbaud, C., Kahane, R., Allen, C., and Prior, P. 2009. Broad diversity of Ralstonia solanacearum strains in Cameroon. Plant Dis. 29:1123-1130.

63. Vailleau, F., Sartorel, E., Jardinaud, M., Chardon, F., Genin, S., Huguet, T., Gentzbittel, L., and Petitprez, M. 2006. Characterization of the interaction between the bacterial wilt pathogen Ralstonia solanacearum and the model legume plant Medicago truncatula. Mol. Plant Microbe Interact. 20:159-167.

64. Villa, J. E., Tsuchiya, K., Horita, M., Natural, M., Opina, N., and Hyakumachi, M. 2005. Phylogenetic relationship of Ralstonia solanacearum species complex strain from Asia and other continents based on 16s rRNA, endoglucanase, and hrpB gene sequences. J. Gen. Plant Pathol. 71:39-46.

65. Wicker, E., Grassart, L., Coranson-Beaudu, R., Mian, D., Guilbaud, C., Fegan, M., and Prior, P. 2007. Ralstonia solanacearum strains from Martinique (French West Indies) exhibiting a new pathogenic potential. Appl. Environ. Microbiol. 73:6790-6801 (Errantum 75:558, 2009).

66. Wicker, E., Lefeuvre, P., de Cambiaire, J.-C., Lemaire, C., Poussier, S., and Prior, P. 2011. Contrasting recombination patterns and demographic histories of the plant pathogen Ralstonia solanacearum inferred from MLSA. Int. Soc. Sci. 6:961-974.

67. Xu, J., Pan, Z. C., Prior, P., Xu, J. S., Zhang, Z., Zhang, H., Zhang, L. Q., He, L. Y., and Feng, J. 2009. Genetic diversity of Ralstonia solanacearum strains from China. Eur. J. Plant Pathol. 125:641-653.

68. Yabuuchi, E., Kosak, Y., Yano, I., Hotta, H., and Nishiuchi, Y. 1995. Transfer of two Burkholderia and an Alcaligenes species to Ralstonia gen. nov. Proposal of Ralstonia picketti (Ralston, Palleroni and Doudoroff 1973) comb. nov., Ralstonia solanacearum (Smith 1896) comb. nov. and Ralstonia eutropha (Davis 1969) comb. nov. Microbiol. Immunol. 39:897-904

69. Young, J. M. 2008. An overview of bacterial nomenclature with special reference to plant pathogens. Syst. Appl. Microbiol. 31:405-424.

70. Yu, Q., Alvarez, A. M., Moore, P. H., Zee, F., Kim, M. S., de Silva, A., Hepperly, P. R., and Ming, R. 2003. Molecular diversity of Ralstonia solanacearum isolated from ginger in Hawaii. Phytopathology 93:11241130.

71. Zhaxybayeva, O., and Gogarten, J. P. 2002. Bootstrap, Bayesian probability and maximum likelihood mapping: Exploring new tools for comparative genome analyses. BMC Genomics 3:4

72. Zwickl, D. J., and Hillis, D. M. 2002. Increased taxon sampling greatly reduces phylogenetic error. Syst. Biol. 51:588-598. 